\title{
Polarization for pair annihilation in strong magnetic fields
}

\author{
L. Semionova ${ }^{1}$ and D. Leahy ${ }^{2}$ \\ 1 Department of Physics, Universidad National, Heredia, 86-3000 Costa Rica \\ 2 Department of Physics and Astronomy, University of Calgary, Alberta, T2N 1N4, Canada
}

Received November 18, 1999; accepted March 16, 2000

\begin{abstract}
We derive the cross-section for one-quantum annihilation of polarized electrons and positrons. The electron and positron can occupy arbitrary Landau levels and have non-zero momentum parallel to the field, and the cross-section is calculated for any polarization of the radiation. When we take zero parallel momentum for the electron and sum over photon polarization we obtain results which agree with the existing calculation of Wunner et al. (1986).
\end{abstract}

Key words: elementary particles — magnetic fields plasmas - polarization — radiation mechanism: thermal

\section{Introduction}

We analyse the annihilation of an electron and a positron producing emission of one photon in a strong magnetic field. It is likely that this annihilation process plays an important role in understanding the radiation mechanism in pulsars and in $\gamma$-ray burst events. With the observational discovery of magnetars (Vasisht \& Gotthelf 1997; Kouveliotou et al. 1999), superstrong magnetic fields, in the range $\sim 10^{13}-10^{15}$ Gauss, are likely to exist, and calculations valid for such strong fields are of great interest.

This process has been considered by previous authors. One photon pair annihilation was first studied by Klepikov (1954), who evaluated the lifetime of highly relativistic $\mathrm{e}^{+}-\mathrm{e}^{-}$beams traversing a magnetic field. Daugherty $\&$ Bussard (1980) obtained the cross-section $\sigma_{1 \gamma}$ and $\sigma_{2 \gamma}$ for the case that electron and positron are in the Landau ground state. They analyzed the cross section for Gaussian distributions of parallel momentum. Harding (1986) studied the one-photon annihilation rate allowing electron and positron to be in excited Landau states. Wunner et al. (1986) considered the effect of electron and positron polarization, and found that the dominant annihilation rate is for the case that the electron spin is

Send offprint requests to: L. Semionova opposite to and the positron spin parallel to the field. Kaminker et al. (1987) studied the two-photon pair annihilation process for the case that the electron and positron are at rest and in the Landau ground state. They found that the magnetic field broadens the spectra and leads to asymmetry in the line around $m c^{2}$ and that the angular distribution depends on magnetic field, $B$, and photon energy, $\omega$, with the peak emission perpendicular to the field. They argue that the annihilation radiation is linearly polarized, the degree and orientation depending on $B$, emission angle to the field, $\theta$, and $\omega$. The polarization may reach tens of percent, even for comparatively small fields $B=0.1 \times B_{\mathrm{cr}}$. The critical value of the magnetic field is defined as $B_{\mathrm{cr}}=\frac{m^{2} c^{3}}{e \hbar}=4.41410^{13}$ Gauss. The polarization is large enough to be detected. However, detectors capable of detecting polarization have not yet been flown in space (although some are planned).

Other emission processes in strong magnetic fields include one and two photon emission (bremsstrahlung) by electrons. The first order (one photon) emission process is discussed by Harding \& Preece (1987) Latal (1986), Semionova (1983), Herold et al. (1982), White (1978) and White (1974). Bussard et al. (1986) calculate first and second order Compton scattering, in which there is one photon in both the initial and final states, in the weak field limit $\left(B<<B_{\mathrm{cr}}\right.$ ). Semionova \& Leahy (1999) calculate the second order emission process, for the different photon polarizations and electron spins, and for any value of magnetic field.

The purpose of this paper is to study the polarization of the annihilation radiation by generalizing previous calculations. The calculation is valid for non-zero values of the parallel momentum of the electron or positron and also valid for any value of magnetic field. To make this possible we use the wave functions of Sokolov \& Ternov (1983) (for details see the discussion in Graziani 1983 and Semionova \& Leahy 1999). We consider the different cases of electron and positron spin and of Landau level, and also calculate cross-sections for the different photon polarizations. 


\section{Transition rates}

A single photon of energy $\omega>2 m / \sin \theta$, with $\theta$ the photon emission angle with respect to the magnetic field, may be produced by electron - positron annihilation in a strong magnetic field. This first order process is permitted in the presence of an external magnetic field, which can absorb the transverse momentum difference between the initial and final particles.

The S-matrix element for this process may be written in the form:

$S_{f i}=(-i e) \int_{-\infty}^{\infty} \mathrm{d} t \int \mathrm{d}^{3} y \bar{\Psi}_{f}(y) \gamma_{\mu} A^{\mu}(y) \Psi_{i}(y)$

with: $A^{\mu}=\epsilon^{\mu} /(2 \omega V)^{1 / 2} \mathrm{e}^{i k y} . \Psi_{f}(y)$ and $\Psi_{i}(y)$ are the wave functions for positron and electron, respectively. We used the wave functions for $\mathrm{e}^{-}$and $\mathrm{e}^{+}$defined by Sokolov \& Ternov (1983).

The photon has four possible polarizations to consider. The two linear polarizations are defined by the unit vectors:

$$
\begin{aligned}
& \hat{\varepsilon}^{(1)}=-\cos \theta \cos \phi \cdot \hat{i}-\cos \theta \sin \phi \cdot \hat{j}+\sin \theta \cdot \hat{k} \\
& \hat{\varepsilon}^{(2)}=\sin \phi \cdot \hat{i}-\cos \phi \cdot \hat{j}
\end{aligned}
$$

where the photon momentum vector is given by:

$\boldsymbol{k}=\omega(\sin \theta \cos \phi \cdot \hat{i}+\sin \theta \sin \phi \cdot \hat{j}+\cos \theta \cdot \hat{k})$.

The unit vectors $\hat{\varepsilon}^{(1)}, \hat{\varepsilon}^{(2)}$ and $\hat{k}$ form a right handed triad: $\hat{\varepsilon}^{(1)} \times \hat{\varepsilon}^{(2)}=\hat{k}$.

The two circular polarizations (right-handed and lefthanded respectively) are given by:

$\hat{\varepsilon}_{ \pm}=\mp \frac{1}{\sqrt{2}}\left(\hat{\varepsilon}^{(1)} \pm i \hat{\varepsilon}^{(2)}\right)$.

The energies for $\mathrm{e}^{-}(E)$ and $\mathrm{e}^{+}\left(E^{\prime}\right)$ are:

$\left.\left.E=\left[m^{2}+p_{z}{ }^{2}+4 N \gamma\right)\right]^{1 / 2} ; E^{\prime}=\left[m^{2}+p_{z}^{\prime 2}+4 N^{\prime} \gamma\right)\right]^{1 / 2}$.

Here $p_{z}$ is the longitudinal momentum for $\mathrm{e}^{-}, p_{z}^{\prime}$ is the longitudinal momentum for $\mathrm{e}^{+}, N=0,1,2, \ldots$ is the Landau level of $\mathrm{e}^{-}, N^{\prime}$ is the Landau level of $\mathrm{e}^{+}$, and $\gamma=\frac{e B}{2 c \hbar}$. In natural units $(c=\hbar=1)$, which we adopt hereafter, $\gamma=\frac{e B}{2}$.

The probability for the annihilation process is:

$$
\begin{aligned}
& \left|S_{f i}\right|^{2}=\frac{\alpha(2 \pi)^{4} T I_{s, s^{\prime}}{ }^{2}\left(\omega_{\perp}{ }^{2} / 2 e B\right)}{\omega L_{x} L_{y}{ }^{2} L_{z}{ }^{2} 16 E^{\prime} E_{\mathrm{o}}{ }^{\prime} E E_{\mathrm{o}}} \delta\left(p_{z}+p_{z}{ }^{\prime}-\omega_{z}\right) \\
& \delta\left(p_{y}+p_{y}{ }^{\prime}-\omega_{y}\right) \delta\left(E+E^{\prime}-\omega\right)\left|N_{\mathrm{ann}}\right|^{2}
\end{aligned}
$$

with:

$$
\begin{aligned}
& \left|N_{\mathrm{ann}}\right|^{2}=\left[\varepsilon^{(\lambda)} \varepsilon_{z}{ }^{(\lambda)}{ }_{z}^{*}\left(E^{\prime} E+p_{z}{ }^{\prime} p_{z}-r r^{\prime} E_{\mathrm{o}}{ }^{\prime} E_{\mathrm{o}}\right)\right. \\
& {\left[\left(E_{\mathrm{O}}{ }^{\prime}-r^{\prime} m\right)\left(E_{\mathrm{O}}+r m\right) I_{N-1, N^{\prime}-1}{ }^{2}\left(\omega_{\perp}{ }^{2} / 2 e B\right)\right.} \\
& \left.+\left(E_{\mathrm{o}}{ }^{\prime}+r^{\prime} m\right)\left(E_{\mathrm{o}}-r m\right) I_{N, N^{\prime}}{ }^{2}\left(\omega_{\perp}{ }^{2} / 2 e B\right)\right] \\
& +r r^{\prime} \sqrt{2 e B N}\left(p_{z} E_{\mathrm{O}}{ }^{\prime}+r r^{\prime} p_{z}{ }^{\prime} E_{\mathrm{o}}\right) \\
& {\left[\left(E_{\mathrm{O}}{ }^{\prime}-r^{\prime} m\right) I_{N-1, N^{\prime}-1}\left(\omega_{\perp}{ }^{2} / 2 e B\right) I_{N, N^{\prime}-1}\left(\omega_{\perp}{ }^{2} / 2 e B\right)\right.} \\
& \left(\varepsilon^{(\lambda)} \varepsilon^{(\lambda)}{ }^{*} \mathrm{e}^{-i \phi}+\varepsilon^{(\lambda)}{ }_{\varepsilon^{(\lambda)}}{ }^{*} \mathrm{e}^{i \phi}\right) \\
& +\left(E_{\mathrm{o}}{ }^{\prime}+r^{\prime} m\right) I_{N-1, N^{\prime}}\left(\omega_{\perp}{ }^{2} / 2 e B\right) I_{N, N^{\prime}}\left(\omega_{\perp}{ }^{2} / 2 e B\right)
\end{aligned}
$$

\begin{tabular}{|c|c|c|c|c|}
\hline & $\lambda=1$ & $\lambda=2$ & $\lambda=+$ & $\lambda=-$ \\
\hline$(\lambda)_{-}$ & & $\begin{array}{l}-i \mathrm{e}^{i \phi} \\
i \mathrm{e}^{-i \phi}\end{array}$ & $\begin{array}{l}-(1-\cos \theta) \mathrm{e}^{-i} / \sqrt{2} \\
(1+\cos \theta) \mathrm{e}^{-i \phi} / \sqrt{2}\end{array}$ & $\begin{array}{l}-(1+\cos \theta) \mathrm{e}^{i \phi} / \sqrt{2} \\
(1-\cos \theta) \mathrm{e}^{-i \phi} / \sqrt{2}\end{array}$ \\
\hline
\end{tabular}

$$
\begin{aligned}
& \left.\left(\varepsilon^{(\lambda)}+\varepsilon^{(\lambda)} z^{*} \mathrm{e}^{-i \phi}+\varepsilon^{(\lambda)} \varepsilon^{(\lambda)}+{ }^{*} \mathrm{e}^{i \phi}\right)\right] \\
& -\sqrt{2 e B N^{\prime}}\left(-p_{z} E_{\mathrm{o}}{ }^{\prime}-r r^{\prime} p_{z}{ }^{\prime} E_{\mathrm{o}}\right) \\
& {\left[\left(E_{\mathrm{o}}+r m\right) I_{N-1, N^{\prime}-1}\left(\omega_{\perp}^{2} / 2 e B\right) I_{N-1, N^{\prime}}\left(\omega_{\perp}^{2} / 2 e B\right)\right.} \\
& \left(\varepsilon^{(\lambda)}{ }_{z} \varepsilon^{(\lambda)}+{ }^{*} \mathrm{e}^{i \phi}+\varepsilon^{(\lambda)}+\varepsilon^{(\lambda)}{ }_{z}^{*} \mathrm{e}^{-i \phi}\right) \\
& +\left(E_{\mathrm{O}}-r m\right) I_{N, N^{\prime}-1}\left(\omega_{\perp}^{2} / 2 e B\right) I_{N, N^{\prime}}\left(\omega_{\perp}^{2} / 2 e B\right) \\
& \left.\left(\varepsilon^{(\lambda)} \varepsilon^{(\lambda)}{ }_{z}^{*} \mathrm{e}^{i \phi}+\varepsilon^{(\lambda)}{ }_{z} \varepsilon^{(\lambda)}{ }_{-}^{*} \mathrm{e}^{-i \phi}\right)\right] \\
& -4 e B \sqrt{N N^{\prime}} \varepsilon^{(\lambda)} \varepsilon^{(\lambda)}{ }_{z}{ }^{*}\left(-r r^{\prime} E^{\prime} E-r r^{\prime} p_{z}{ }^{\prime} p_{z}+E_{\mathrm{o}}{ }^{\prime} E_{\mathrm{o}}\right) \\
& I_{N-1, N^{\prime}-1}\left(\omega_{\perp}^{2} / 2 e B\right) I_{N, N^{\prime}}\left(\omega_{\perp}^{2} / 2 e B\right) \\
& +\left(E^{\prime} E-p_{z}{ }^{\prime} p_{z}+r r^{\prime} E_{\mathrm{o}}{ }^{\prime} E_{\mathrm{o}}\right)\left[\varepsilon^{(\lambda)}{ }_{-} \varepsilon^{(\lambda)}{ }_{-}{ }^{*}\left(E_{\mathrm{o}}{ }^{\prime}-r^{\prime} m\right)\right. \\
& \left(E_{\mathrm{o}}-r m\right) I_{N, N^{\prime}-1}^{2}\left(\omega_{\perp}^{2} / 2 e B\right) \\
& +\varepsilon^{(\lambda)}+\varepsilon^{(\lambda)}+{ }^{*}\left(E_{\mathrm{o}}{ }^{\prime}+r^{\prime} m\right)\left(E_{\mathrm{o}}+r m\right) I_{N-1, N^{\prime}}{ }^{2} \\
& \left.\left(\omega_{\perp}^{2} / 2 e B\right)\right] \\
& +2 e B \sqrt{N N^{\prime}}\left(r r^{\prime} E^{\prime} E-r r^{\prime} p_{z}{ }^{\prime} p_{z}+E_{\mathrm{o}}{ }^{\prime} E_{\mathrm{o}}\right) \\
& I_{N, N^{\prime}-1}\left(\omega_{\perp}^{2} / 2 e B\right) I_{N-1, N^{\prime}}\left(\omega_{\perp}^{2} / 2 e B\right) \\
& \left.\left(\varepsilon^{(\lambda)} \varepsilon^{(\lambda)}+{ }^{*} \mathrm{e}^{i 2 \phi}+\varepsilon^{(\lambda)}+\varepsilon^{(\lambda)}{ }^{*} \mathrm{e}^{-i 2 \phi}\right)\right]
\end{aligned}
$$

with $E_{\mathrm{o}}=\left(m^{2}+2 N e B\right)^{1 / 2}, E_{\mathrm{o}}{ }^{\prime}=\left(m^{2}+2 N^{\prime} e B\right)^{1 / 2}$, and $\varepsilon^{(\lambda)}{ }_{ \pm}=\varepsilon^{(\lambda)}{ }_{x} \pm i \varepsilon^{(\lambda)}{ }_{y}$. Formulae for $\varepsilon^{(\lambda)} \pm$ are given explicity in Table 1 . The polarization of the electron spin is $r= \pm 1$, except for $N=0$ which has $r=-1$ only. The polarization of the positron spin is $r^{\prime}= \pm 1$, except for $N^{\prime}=0$ which has $r^{\prime}=+1$ only.

Table 1. $\varepsilon^{(\lambda)} \pm$ for different photon polarizations

The Laguerre function is:

$I_{N, R}(x)=\sqrt{R ! / N !} \mathrm{e}^{-x / 2} x^{(N-R) / 2} L_{R}{ }^{N-R}(x)$.

If we sum over radial quantum number $s^{\prime}$ :

$\sum_{s^{\prime}=0}^{\infty} I_{s, s^{\prime}}{ }^{2}(x)=1$.

However, the sum over $\mathrm{s}$ is limited to some maximum value $s_{\text {max }}$ (see Sokolov \& Ternov 1983), so

$\sum_{s=0}^{s_{\max }} \sum_{s^{\prime}=0}^{\infty} I_{s, s^{\prime}}{ }^{2}\left(\omega_{\perp}^{2} / 2 e B\right)=\frac{m^{2} B}{2 \pi B_{\mathrm{cr}}} L^{2}$.

The evaluation of the probability for the 1 photon annihilation process using Sokolov's spinors is different with respect to the evaluation using Herold's spinors (Herold 1979) as follows. We obtain one more term $I_{s, s^{\prime}}{ }^{2}\left(\omega_{\perp}^{2} / 2 e B\right)$ and only two laws of conservation:

$p_{z}+p_{z}{ }^{\prime}=\omega_{z} \quad E+E^{\prime}=\omega$.

In comparison Wunner et al. (1986) has one more conservation law $p_{y}+p_{y}{ }^{\prime}=\omega_{y}$. 
The transition rate is given by:

$$
\begin{aligned}
& W=\lim _{T \rightarrow \infty} \frac{1}{T} \sum_{\text {final states }} \sum_{\text {initial states }}\left|S_{f i}\right|^{2} \\
& =\lim _{T \rightarrow \infty} \frac{1}{T} \iint \frac{\alpha 2 \pi \omega \frac{B_{\mathrm{cr}}}{B} T \mathrm{~d} \omega \mathrm{d} \Omega}{L_{x} L_{y} L_{z} m^{2} 16 E^{\prime} E_{\mathrm{o}}{ }^{\prime} E E_{\mathrm{o}}}\left|N_{\text {ann }}\right|^{2}
\end{aligned}
$$

with $p_{y}+p_{y}{ }^{\prime}=\omega_{y}$ and $E+E^{\prime}=\omega . \alpha$ denotes the fine structure constant $\left(\alpha=\mathrm{e}^{2} / \hbar c\right.$ or $\mathrm{e}^{2}$ in natural units). The differential transition rate is:

$\mathrm{d} W_{r . r^{\prime}}{ }^{N, N^{\prime}}(\omega, \lambda)=\alpha(\pi / 2) \frac{m}{V} \frac{B_{\mathrm{cr}}}{m^{3} B} \frac{\mathrm{d} \phi}{2 E^{\prime} E_{\mathrm{o}}{ }^{\prime} E E_{\mathrm{o}}}\left|N_{\text {ann }}\right|^{2}$

Restricting to the case of zero longitudinal momentum for the electron $\left(p_{z}=0\right)$, we obtain:

$$
\begin{aligned}
& \mathrm{d} W_{r . r^{\prime}}{ }^{N, N^{\prime}}(\omega, \lambda)=\alpha(\pi / 2) \frac{m}{V} \frac{B_{\mathrm{cr}}}{m^{3} B} \frac{\mathrm{d} \phi}{2 E^{\prime} E_{\mathrm{o}}{ }^{\prime} E_{\mathrm{o}}} \\
& {\left[\left(E_{\mathrm{o}}-r m\right)\left(E_{\mathrm{o}}{ }^{\prime}+r^{\prime} m\right)\left(E^{\prime}-r r^{\prime} E_{\mathrm{O}}{ }^{\prime}\right) I_{N, N^{\prime}}{ }^{2}\right.} \\
& \left(\omega_{\perp}^{2} / 2 e B\right) \varepsilon^{(\lambda)} \varepsilon^{(\lambda)}{ }_{z}^{*} \\
& +\left(E_{\mathrm{o}}+r m\right)\left(E_{\mathrm{o}}{ }^{\prime}+r^{\prime} m\right)\left(E^{\prime}+r r^{\prime} E_{\mathrm{o}}{ }^{\prime}\right) I_{N-1, N^{\prime}}{ }^{2} \\
& \left(\omega_{\perp}^{2} / 2 e B\right) \varepsilon^{(\lambda)}+\varepsilon^{(\lambda)}+{ }^{*} \\
& +\left(E_{\mathrm{o}}+r m\right)\left(E_{\mathrm{o}}{ }^{\prime}-r^{\prime} m\right)\left(E^{\prime}-r r^{\prime} E_{\mathrm{o}}{ }^{\prime}\right) I_{N-1, N^{\prime}-1}{ }^{2} \\
& \left(\omega_{\perp}^{2} / 2 e B\right) \varepsilon^{(\lambda)} \varepsilon^{(\lambda)}{ }_{z}^{*} \\
& +\left(E_{\mathrm{o}}-r m\right)\left(E_{\mathrm{o}}{ }^{\prime}-r^{\prime} m\right)\left(E^{\prime}+r r^{\prime} E_{\mathrm{o}}{ }^{\prime}\right) I_{N, N^{\prime}-1}{ }^{2} \\
& \left(\omega_{\perp}^{2} / 2 e B\right) \varepsilon^{(\lambda)}-\varepsilon^{(\lambda)}{ }^{*} \\
& +\sqrt{2 e B N} p_{z \mathrm{o}}{ }^{\prime}\left[\left(E_{\mathrm{o}}{ }^{\prime}+r^{\prime} m\right) I_{N, N^{\prime}}\left(\omega_{\perp}{ }^{2} / 2 e B\right) I_{N-1, N^{\prime}}\right. \\
& \left(\omega_{\perp}^{2} / 2 e B\right) \\
& \left(\varepsilon^{(\lambda)}+\varepsilon^{(\lambda)}{ }_{z}^{*} \mathrm{e}^{-i \phi}+\varepsilon^{(\lambda)}{ }_{z} \varepsilon^{(\lambda)}+{ }^{*} \mathrm{e}^{i \phi}\right) \\
& +\left(E_{\mathrm{o}}{ }^{\prime}-r / m\right) I_{N-1, N^{\prime}-1} \\
& \left(\omega_{\perp}^{2} / 2 e B\right) I_{N, N^{\prime}-1}\left(\omega_{\perp}^{2} / 2 e B\right) \\
& \left.\left(\varepsilon^{(\lambda)} \varepsilon_{z} \varepsilon^{(\lambda)}-{ }^{*} \mathrm{e}^{-i \phi}+\varepsilon^{(\lambda)}-\varepsilon^{(\lambda)}{ }_{z}^{*} \mathrm{e}^{i \phi}\right)\right] \\
& +r r^{\prime} \sqrt{2 e B N^{\prime}} p_{z \mathrm{o}}{ }^{\prime}\left[\left(E_{\mathrm{o}}+r m\right) I_{N-1, N^{\prime}-1}\right. \\
& \left(\omega_{\perp}^{2} / 2 e B\right) I_{N-1, N^{\prime}}\left(\omega_{\perp}^{2} / 2 e B\right) \\
& \left(\varepsilon^{(\lambda)}{ }_{+} \varepsilon^{(\lambda)}{ }_{z}{ }^{*} \mathrm{e}^{-i \phi}+\varepsilon^{(\lambda)}{ }_{z} \varepsilon^{(\lambda)}+{ }^{*} \mathrm{e}^{i \phi}\right) \\
& +\left(E_{\mathrm{O}}-r m\right) I_{N, N^{\prime}}\left(\omega_{\perp}{ }^{2} / 2 e B\right) I_{N, N^{\prime}-1}\left(\omega_{\perp}{ }^{2} / 2 e B\right) \\
& \left.\left(\varepsilon^{(\lambda)} \varepsilon^{(\lambda)}-{ }^{*} \mathrm{e}^{-i \phi}+\varepsilon^{(\lambda)}-\varepsilon^{(\lambda)} z^{*} \mathrm{e}^{i \phi}\right)\right] \\
& +2 e B \sqrt{N N^{\prime}}\left[\left(E_{\mathrm{o}}{ }^{\prime}+r r^{\prime} E^{\prime}\right) I_{N-1, N^{\prime}}\left(\omega_{\perp}{ }^{2} / 2 e B\right) I_{N, N^{\prime}-1}\right. \\
& \left(\omega_{\perp}^{2} / 2 e B\right) \\
& \left.\left(\varepsilon^{(\lambda)} \varepsilon^{(\lambda)}+{ }^{*} \mathrm{e}^{i 2 \phi}+\varepsilon^{(\lambda)}+\varepsilon^{(\lambda)}{ }^{*} \mathrm{e}^{-i 2 \phi}\right)\right] \\
& +2 r r^{\prime}\left(E^{\prime}-r r^{\prime} E_{\mathrm{o}}{ }^{\prime}\right) I_{N, N^{\prime}}\left(\omega_{\perp}{ }^{2} / 2 e B\right) I_{N-1, N^{\prime}-1} \\
& \left.\left.\left(\omega_{\perp}^{2} / 2 e B\right) \varepsilon^{(\lambda)}{ }_{z} \varepsilon^{(\lambda)}{ }^{*}\right]\right] \text {. }
\end{aligned}
$$

Here $p_{z \mathrm{o}}{ }^{\prime}$ is the longitudinal momentum of the positron when the electron longitudinal momentum is zero.

The energy of the annihilation radiation is given by:

$$
\begin{aligned}
\omega & =\frac{\left(E-p_{z} \cos \theta\right)+\sqrt{\left(E-p_{z} \cos \theta\right)^{2}-4 \sin ^{2} \theta \gamma\left(N-N^{\prime}\right)}}{\sin ^{2} \theta} \\
& =\frac{\left(E-p_{z} \cos \theta\right)}{\sin ^{2} \theta}\left[1+\sqrt{1-\frac{2\left(N-N^{\prime}\right) m^{2}\left(B / B_{\mathrm{cr}}\right) \sin ^{2} \theta}{\left(E-p_{z} \cos \theta\right)^{2}}}\right] \cdot
\end{aligned}
$$

This follows from the two conservation laws $p_{z}+p_{z}{ }^{\prime}=$ $\omega \cos \theta$ and $E+E^{\prime}=\omega$.

The coefficients above related to the polarization of the photon are evaluated and listed in Table 2. By averaging over polarization of the positron, the differential transition rate for a polarized electron $(r= \pm 1)$ and unpolarized positron (if $p_{z} \neq 0$ ) is:

$$
\begin{aligned}
& \mathrm{d} W_{r}{ }^{N, N^{\prime}}(\omega, \lambda)=\frac{\alpha \pi m B_{\mathrm{cr}}}{V m^{3} B} \frac{\mathrm{d} \phi}{2 E^{\prime} E E_{\mathrm{o}}} \frac{\left(1+\delta_{N^{\prime}, 0}\right)}{2} \\
& {\left[\varepsilon ^ { ( \lambda ) } { } _ { z } \varepsilon ^ { ( \lambda ) } { } _ { z } ^ { * } \left[\left(E_{\mathrm{o}}+r m\right)\left(E^{\prime} E+p_{z}{ }^{\prime} p_{z}+r E_{\mathrm{o}} m\right) I_{N-1, N^{\prime}-1}{ }^{2}\right.\right.} \\
& \left(\omega_{\perp}^{2} / 2 e B\right) \\
& \left.+\left(E_{\mathrm{o}}-r m\right)\left(E^{\prime} E+p_{z}{ }^{\prime} p_{z}-r E_{\mathrm{o}} m\right) I_{N, N^{\prime}}{ }^{2}\left(\omega_{\perp}{ }^{2} / 2 e B\right)\right] \\
& +\sqrt{2 e B N}\left[\left(p_{z}{ }^{\prime} E_{\mathrm{o}}-r p_{z} m\right) I_{N-1, N^{\prime}-1}\left(\omega_{\perp}{ }^{2} / 2 e B\right) I_{N, N^{\prime}-1}\right. \\
& \left(\omega_{\perp}^{2} / 2 e B\right) \\
& \left(\varepsilon^{(\lambda)}{ }_{z} \varepsilon^{(\lambda)}{ }_{-}^{*} \mathrm{e}^{-i \phi}+\varepsilon^{(\lambda)} \varepsilon_{-}^{(\lambda)}{ }_{z}^{*} \mathrm{e}^{i \phi}\right) \\
& +\left(p_{z}{ }^{\prime} E_{\mathrm{o}}+r p_{z} m\right) I_{N-1, N^{\prime}}\left(\omega_{\perp}{ }^{2} / 2 e B\right) I_{N, N^{\prime}}\left(\omega_{\perp}{ }^{2} / 2 e B\right) \\
& \left.\left(\varepsilon^{(\lambda)}+\varepsilon^{(\lambda)}{ }_{z}^{*} \mathrm{e}^{-i \phi}+\varepsilon^{(\lambda)} \varepsilon^{(\lambda)}+{ }^{*} \mathrm{e}^{i \phi}\right)\right] \\
& +\sqrt{2 e B N^{\prime}} p_{z}\left[\left(E_{\mathrm{O}}+r m\right) I_{N-1, N^{\prime}-1}\left(\omega_{\perp}^{2} / 2 e B\right) I_{N-1, N^{\prime}}\right. \\
& \left(\omega_{\perp}^{2} / 2 e B\right) \\
& \left(\varepsilon^{(\lambda)}{ }_{z} \varepsilon^{(\lambda)}+{ }^{*} \mathrm{e}^{i \phi}+\varepsilon^{(\lambda)}+\varepsilon^{(\lambda)}{ }_{z}^{*} \mathrm{e}^{-i \phi}\right) \\
& +\left(E_{\mathrm{O}}-r m\right) I_{N, N^{\prime}-1}\left(\omega_{\perp}^{2} / 2 e B\right) I_{N, N^{\prime}}\left(\omega_{\perp}^{2} / 2 e B\right) \\
& \left.\left(\varepsilon^{(\lambda)} \varepsilon^{(\lambda)} z^{*} \mathrm{e}^{i \phi}+\varepsilon^{(\lambda)} z^{(\lambda)}{ }^{*} \mathrm{e}^{-i \phi}\right)\right] \\
& -2 e B \sqrt{N N^{\prime}} E_{\mathrm{o}}\left[2 \varepsilon^{(\lambda)}{ }_{z} \varepsilon^{(\lambda)}{ }_{z}^{*} I_{N-1, N^{\prime}-1}\left(\omega_{\perp}{ }^{2} / 2 e B\right) I_{N, N^{\prime}}\right. \\
& \left(\omega_{\perp}^{2} / 2 e B\right) \\
& -I_{N, N^{\prime}-1}\left(\omega_{\perp}^{2} / 2 e B\right) I_{N-1, N^{\prime}}\left(\omega_{\perp}^{2} / 2 e B\right) \\
& \left.\left(\varepsilon^{(\lambda)}-\varepsilon^{(\lambda)}+{ }^{*} \mathrm{e}^{i 2 \phi}+\varepsilon^{(\lambda)}+\varepsilon^{(\lambda)}{ }^{*} \mathrm{e}^{-i 2 \phi}\right)\right] \\
& +\left[\left(E_{\mathrm{O}}-r m\right)\left(E^{\prime} E-p_{z}{ }^{\prime} p_{z}-r E_{\mathrm{O}} m\right) \varepsilon^{(\lambda)}{ }_{-} \varepsilon^{(\lambda)}{ }_{-}{ }^{*} I_{N, N^{\prime}-1}{ }^{2}\right. \\
& \left(\omega_{\perp}^{2} / 2 e B\right) \\
& +\left(E_{\mathrm{o}}+r m\right)\left(E^{\prime} E-p_{z}{ }^{\prime} p_{z}+r E_{\mathrm{o}} m\right) \varepsilon^{(\lambda)}+\varepsilon^{(\lambda)}{ }^{*}{ }^{*} I_{N-1, N^{\prime}}{ }^{2} \\
& \left.\left.\left(\omega_{\perp}^{2} / 2 e B\right)\right]\right] \text {. }
\end{aligned}
$$

For the case of zero electron longitudinal momentum $\left(p_{z}=0\right)$ this reduces to:

$$
\begin{aligned}
& \mathrm{d} W_{\mp}{ }^{N, N^{\prime}}(\omega, \lambda)=\frac{\alpha \pi m B_{\mathrm{cr}}}{V m^{3} B} \frac{\mathrm{d} \phi}{2 E^{\prime} E_{\mathrm{o}}} \frac{\left(1+\delta_{N^{\prime}, 0}\right)}{2} \\
& {\left[\varepsilon ^ { ( \lambda ) } { } _ { z } \varepsilon ^ { ( \lambda ) } { } _ { z } ^ { * } \left[\left(E_{\mathrm{O}} \mp m\right)\left(E^{\prime} \mp m\right) I_{N-1, N^{\prime}-1}{ }^{2}\left(\omega_{\perp}{ }^{2} / 2 e B\right)\right.\right.} \\
& \left.+\left(E_{\mathrm{o}} \pm m\right)\left(E^{\prime} \pm m\right) I_{N, N^{\prime}}^{2}\left(\omega_{\perp}^{2} / 2 e B\right)\right] \\
& +p_{z \mathrm{o}}{ }^{\prime} \sqrt{2 e B N}\left[I_{N-1, N^{\prime}-1}\left(\omega_{\perp}{ }^{2} / 2 e B\right) I_{N, N^{\prime}-1}\left(\omega_{\perp}{ }^{2} / 2 e B\right)\right. \\
& \left(\varepsilon^{(\lambda)} z^{(\lambda)}{ }^{*} \mathrm{e}^{-i \phi}+\varepsilon^{(\lambda)}-\varepsilon^{(\lambda)} z^{*} \mathrm{e}^{i \phi}\right) \\
& +I_{N-1, N^{\prime}}\left(\omega_{\perp}^{2} / 2 e B\right) I_{N, N^{\prime}}\left(\omega_{\perp}^{2} / 2 e B\right) \\
& \left.\left(\varepsilon^{(\lambda)}+\varepsilon^{(\lambda)} z^{*} \mathrm{e}^{-i \phi}+\varepsilon^{(\lambda)} \varepsilon^{(\lambda)}+{ }^{*} \mathrm{e}^{i \phi}\right)\right] \\
& -2 e B \sqrt{N N^{\prime}}\left[2 \varepsilon^{(\lambda)}{ }_{z} \varepsilon^{(\lambda)}{ }_{z}^{*} I_{N-1, N^{\prime}-1}\left(\omega_{\perp}{ }^{2} / 2 e B\right) I_{N, N^{\prime}}\right. \\
& \left(\omega_{\perp}^{2} / 2 e B\right) \\
& -I_{N, N^{\prime}-1}\left(\omega_{\perp}^{2} / 2 e B\right) I_{N-1, N^{\prime}}\left(\omega_{\perp}^{2} / 2 e B\right) \\
& \left.\left(\varepsilon^{(\lambda)}{ }_{-} \varepsilon^{(\lambda)}+{ }^{*} \mathrm{e}^{i 2 \phi}+\varepsilon^{(\lambda)}+\varepsilon^{(\lambda)}{ }_{-}^{*} \mathrm{e}^{-i 2 \phi}\right)\right] \\
& +\left[\left(E_{\mathrm{o}} \pm m\right)\left(E^{\prime} \pm m\right) \varepsilon^{(\lambda)}-\varepsilon^{(\lambda)}{ }_{-}^{*} I_{N, N^{\prime}-1}{ }^{2}\left(\omega_{\perp}{ }^{2} / 2 e B\right)\right.
\end{aligned}
$$


Table 2. Coefficients related to the polarization of the photon

\begin{tabular}{lllll}
\hline Coefficient & $\sum_{\lambda}$ & $\lambda=1$ & $\lambda=2$ & $\lambda= \pm$ \\
\hline$\varepsilon^{(\lambda)}{ }_{-} \varepsilon^{(\lambda)}{ }^{*}$ & $1+\cos ^{2} \theta$ & $\cos ^{2} \theta$ & 1 & $(1 \pm \cos \theta)^{2} / 2$ \\
$\varepsilon^{(\lambda)}{ }^{2} \varepsilon^{(\lambda)}{ }^{*}$ & $-\sin (2 \theta) \mathrm{e}^{-i \phi} / 2$ & $-\sin (2 \theta) \mathrm{e}^{-i \phi} / 2$ & 0 & $\mp(1 \pm \cos \theta) \sin \theta \mathrm{e}^{-i \phi} / 2$ \\
$\varepsilon^{(\lambda)}{ }_{-} \varepsilon^{(\lambda)}{ }^{*}$ & $-\sin ^{2} \theta \mathrm{e}^{-i 2 \phi}$ & $\cos ^{2} \theta \mathrm{e}^{-i 2 \phi}$ & $-\mathrm{e}^{-i 2 \phi}$ & $-\sin ^{2} \theta \mathrm{e}^{-i 2 \phi} / 2$ \\
$\varepsilon^{(\lambda)}{ }_{z} \varepsilon^{(\lambda)}{ }^{*}$ & $-\sin (2 \theta) \mathrm{e}^{i \phi} / 2$ & $-\sin (2 \theta) \mathrm{e}^{i \phi} / 2$ & 0 & $\mp(1 \pm \cos \theta) \sin \theta \mathrm{e}^{i \phi} / 2$ \\
$\varepsilon^{(\lambda)}{ }_{z} \varepsilon^{(\lambda)}{ }^{*}$ & $\sin ^{2} \theta$ & $\sin ^{2} \theta$ & 0 & $\left(\sin ^{2} \theta\right) / 2$ \\
$\varepsilon^{(\lambda)}{ }_{z} \varepsilon^{(\lambda)}{ }^{+}{ }^{*}$ & $-\sin (2 \theta) \mathrm{e}^{-i \phi} / 2$ & $-\sin (2 \theta) \mathrm{e}^{-i \phi} / 2$ & 0 & $\pm(1 \mp \cos \theta) \sin \theta \mathrm{e}^{-i \phi} / 2$ \\
$\varepsilon^{(\lambda)}{ }_{+} \varepsilon^{(\lambda)}{ }^{*}$ & $-\sin ^{2} \theta \mathrm{e}^{i 2 \phi}$ & $\cos ^{2} \theta \mathrm{e}^{i 2 \phi}$ & $-\mathrm{e}^{i 2 \phi}$ & $-\sin ^{2} \theta \mathrm{e}^{i 2 \phi} / 2$ \\
$\varepsilon^{(\lambda)}{ }_{+} \varepsilon^{(\lambda)}{ }^{*}$ & $-\sin (2 \theta) \mathrm{e}^{i \phi} / 2$ & $-\sin (2 \theta) \mathrm{e}^{i \phi} / 2$ & 0 & $\pm(1 \mp \cos \theta) \sin \theta \mathrm{e}^{i \phi} / 2$ \\
$\varepsilon^{(\lambda)}{ }_{+} \varepsilon^{(\lambda)}{ }_{+}{ }^{*}$ & $1+\cos ^{2} \theta$ & $\cos ^{2} \theta$ & 1 & $(1 \mp \cos \theta)^{2} / 2$ \\
\hline
\end{tabular}

$$
\left.\left.+\left(E_{\mathrm{o}} \mp m\right)\left(E^{\prime} \mp m\right) \varepsilon^{(\lambda)}+\varepsilon^{(\lambda)}+{ }^{*} I_{N-1, N^{\prime}}{ }^{2}\left(\omega_{\perp}^{2} / 2 e B\right)\right]\right]
$$

Alternatively, if we average $\mathrm{d} W_{r \cdot r^{\prime}}{ }^{N, N^{\prime}}(\omega, \lambda)$ over the polarization of the electron, the differential probability for a polarized positron $\left(r^{\prime}= \pm 1\right)$ and an unpolarized electron is obtained (for $p_{z} \neq 0$ ):

$$
\begin{aligned}
& \mathrm{d} W_{r^{\prime}=\mp 1}{ }^{N, N^{\prime}}(\omega, \lambda)=\frac{\alpha \pi m B_{\mathrm{cr}}}{V m^{3} B} \frac{\mathrm{d} \phi}{2 E^{\prime} E E_{\mathrm{o}}{ }^{\prime}} \frac{\left(1+\delta_{N, 0}\right)}{2} \\
& {\left[\varepsilon ^ { ( \lambda ) } { } _ { z } \varepsilon ^ { ( \lambda ) } { } _ { z } { } ^ { * } \left[\left(E_{\mathrm{O}}{ }^{\prime} \pm m\right)\left(E^{\prime} E+p_{z}{ }^{\prime} p_{z} \pm E_{\mathrm{O}}{ }^{\prime} m\right) I_{N-1, N^{\prime}-1}{ }^{2}\right.\right.} \\
& \left(\omega_{\perp}^{2} / 2 e B\right) \\
& +\left(E_{\mathrm{o}}{ }^{\prime} \mp m\right)\left(E^{\prime} E+p_{z}{ }^{\prime} p_{z} \mp E_{\mathrm{o}}{ }^{\prime} m\right) I_{N, N^{\prime}}{ }^{2} \\
& \left.\left(\omega_{\perp}^{2} / 2 e B\right)\right] \\
& +p_{z}{ }^{\prime} \sqrt{2 e B N}\left[\left(E_{\mathrm{o}}{ }^{\prime} \pm m\right) I_{N-1, N^{\prime}-1}\left(\omega_{\perp}{ }^{2} / 2 e B\right) I_{N, N^{\prime}-1}\right. \\
& \left(\omega_{\perp}^{2} / 2 e B\right) \\
& \left(\varepsilon^{(\lambda)} \varepsilon^{(\lambda)}-{ }^{*} \mathrm{e}^{-i \phi}+\varepsilon^{(\lambda)}-\varepsilon^{(\lambda)}{ }_{z}^{*} \mathrm{e}^{i \phi}\right) \\
& +\left(E_{\mathrm{O}}{ }^{\prime} \mp m\right) I_{N-1, N^{\prime}}\left(\omega_{\perp}{ }^{2} / 2 e B\right) I_{N, N^{\prime}}\left(\omega_{\perp}{ }^{2} / 2 e B\right) \\
& \left.\left(\varepsilon^{(\lambda)}+\varepsilon^{(\lambda)} z^{*} \mathrm{e}^{-i \phi}+\varepsilon^{(\lambda)} \varepsilon^{(\lambda)}+{ }^{*} \mathrm{e}^{i \phi}\right)\right] \\
& -\sqrt{2 e B N^{\prime}}\left( \pm p_{z}{ }^{\prime} m-p_{z} E_{\mathrm{o}}{ }^{\prime}\right) I_{N-1, N^{\prime}-1}\left(\omega_{\perp}{ }^{2} / 2 e B\right) I_{N-1, N^{\prime}} \\
& \left(\omega_{\perp}^{2} / 2 e B\right) \\
& \left(\varepsilon^{(\lambda)}{ }_{z} \varepsilon^{(\lambda)}+{ }^{*} \mathrm{e}^{i \phi}+\varepsilon^{(\lambda)}+\varepsilon^{(\lambda)}{ }_{z}{ }^{*} \mathrm{e}^{-i \phi}\right) \\
& +\sqrt{2 e B N^{\prime}}\left( \pm p_{z}{ }^{\prime} m+p_{z} E_{\mathrm{o}}{ }^{\prime}\right) I_{N, N^{\prime}-1}\left(\omega_{\perp}{ }^{2} / 2 e B\right) I_{N, N^{\prime}} \\
& \left(\omega_{\perp}^{2} / 2 e B\right) \\
& \left(\varepsilon^{(\lambda)}{ }_{-} \varepsilon^{(\lambda)}{ }_{z}^{*} \mathrm{e}^{i \phi}+\varepsilon^{(\lambda)} \varepsilon_{z} \varepsilon^{(\lambda)}{ }^{*} \mathrm{e}^{-i \phi}\right) \\
& -4 e B \sqrt{N N^{\prime}} E_{\mathrm{O}}{ }^{\prime}{ }^{(\lambda)}{ }_{z} \varepsilon^{(\lambda)}{ }_{z}{ }^{*} I_{N-1, N^{\prime}-1}\left(\omega_{\perp}{ }^{2} / 2 e B\right) I_{N, N^{\prime}} \\
& \left(\omega_{\perp}^{2} / 2 e B\right) \\
& +\left(E_{\mathrm{o}}{ }^{\prime} \pm m\right)\left(E^{\prime} E-p_{z}{ }^{\prime} p_{z} \pm E_{\mathrm{o}}{ }^{\prime} m\right) \varepsilon^{(\lambda)}-\varepsilon^{(\lambda)}{ }_{-}{ }^{*} I_{N, N^{\prime}-1}{ }^{2} \\
& \left(\omega_{\perp}^{2} / 2 e B\right) \\
& +\left(E_{\mathrm{o}}{ }^{\prime} \mp m\right)\left(E^{\prime} E-p_{z}{ }^{\prime} p_{z} \mp E_{\mathrm{o}}{ }^{\prime} m\right) \varepsilon^{(\lambda)}+\varepsilon^{(\lambda)}+{ }^{*} I_{N-1, N^{\prime}}{ }^{2} \\
& \left(\omega_{\perp}^{2} / 2 e B\right) \\
& +2 e B \sqrt{N N^{\prime}} E_{\mathrm{O}}{ }^{\prime} I_{N, N^{\prime}-1}\left(\omega_{\perp}{ }^{2} / 2 e B\right) I_{N-1, N^{\prime}}\left(\omega_{\perp}{ }^{2} / 2 e B\right) \\
& \left.\left(\varepsilon^{(\lambda)} \varepsilon^{(\lambda)}+{ }^{*} \mathrm{e}^{i 2 \phi}+\varepsilon^{(\lambda)}+\varepsilon^{(\lambda)}{ }^{*} \mathrm{e}^{-i 2 \phi}\right)\right] \text {. }
\end{aligned}
$$

\section{Total annihilation cross-sections}

Integrating the differential rate $\mathrm{d} W$ over the azimuth angle, $\phi$, and dividing the resulting rate by the current density $\left(=\left|p_{z} / E-p_{z}{ }^{\prime} / E^{\prime}\right| / V\right)$ we obtain the one-photon annihilation cross-section (for any $p_{z}$ ):

$$
\begin{aligned}
& \sigma_{r, r^{\prime}}{ }^{N, N^{\prime}}=\frac{\alpha \lambda_{\mathrm{c}}{ }^{2} B_{\mathrm{cr}}}{4 B} \frac{1}{2 p_{z}^{\prime} E_{\mathrm{o}}{ }^{\prime} E E_{\mathrm{o}}} \\
& {\left[\varepsilon^{(\lambda)}{ }_{z} \varepsilon^{(\lambda)}{ }_{z}{ }^{*}\left(E^{\prime} E+p_{z}{ }^{\prime} p_{z}-r r^{\prime} E_{\mathrm{o}}{ }^{\prime} E_{\mathrm{o}}\right)\right.} \\
& {\left[\left(E_{\mathrm{O}}+r m\right)\left(E_{\mathrm{o}}{ }^{\prime}-r^{\prime} m\right) I_{N-1, N^{\prime}-1}{ }^{2}\left(\omega_{\perp}{ }^{2} / 2 e B\right)\right.} \\
& \left.+\left(E_{\mathrm{o}}-r m\right)\left(E_{\mathrm{o}}{ }^{\prime}+r^{\prime} m\right) I_{N, N^{\prime}}{ }^{2}\left(\omega_{\perp}{ }^{2} / 2 e B\right)\right] \\
& +r r^{\prime} \sqrt{2 e B N}\left(p_{z} E_{\mathrm{O}}{ }^{\prime}+r r^{\prime} p_{z}{ }^{\prime} E_{\mathrm{O}}\right) \\
& {\left[\left(E_{\mathrm{o}}{ }^{\prime}-r^{\prime} m\right) I_{N-1, N^{\prime}-1}\left(\omega_{\perp}{ }^{2} / 2 e B\right) I_{N, N^{\prime}-1}\left(\omega_{\perp}{ }^{2} / 2 e B\right)\right.} \\
& \left(\varepsilon^{(\lambda)}{ }_{z} \varepsilon^{(\lambda)}-{ }^{*} \mathrm{e}^{-i \phi}+\varepsilon^{(\lambda)} \varepsilon^{(\lambda)}{ }_{z}{ }^{*} \mathrm{e}^{i \phi}\right) \\
& +\left(E_{\mathrm{O}}{ }^{\prime}+r^{\prime} m\right) I_{N, N^{\prime}}\left(\omega_{\perp}{ }^{2} / 2 e B\right) I_{N-1, N^{\prime}}\left(\omega_{\perp}{ }^{2} / 2 e B\right) \\
& \left.\left(\varepsilon^{(\lambda)}+\varepsilon^{(\lambda)}{ }_{z}^{*} \mathrm{e}^{-i \phi}+\varepsilon^{(\lambda)} \varepsilon^{(\lambda)}+{ }^{*} \mathrm{e}^{i \phi}\right)\right] \\
& -\sqrt{2 e B N^{\prime}}\left(-p_{z} E_{\mathrm{o}}{ }^{\prime}-r r^{\prime} p_{z}{ }^{\prime} E_{\mathrm{o}}\right) \\
& {\left[\left(E_{\mathrm{o}}+r m\right) I_{N-1, N^{\prime}-1}\left(\omega_{\perp}^{2} / 2 e B\right) I_{N-1, N^{\prime}}\left(\omega_{\perp}^{2} / 2 e B\right)\right.} \\
& \left(\varepsilon^{(\lambda)}+\varepsilon^{(\lambda)} z^{*} \mathrm{e}^{-i \phi}+\varepsilon^{(\lambda)}{ }_{z} \varepsilon^{(\lambda)}+{ }^{*} \mathrm{e}^{i \phi}\right) \\
& +\left(E_{\mathrm{O}}-r m\right) I_{N, N^{\prime}}\left(\omega_{\perp}^{2} / 2 e B\right) I_{N, N^{\prime}-1}\left(\omega_{\perp}^{2} / 2 e B\right) \\
& \left.\left(\varepsilon^{(\lambda)} \varepsilon^{(\lambda)} \varepsilon^{*} \mathrm{e}^{-i \phi}+\varepsilon^{(\lambda)}{ }_{-} \varepsilon^{(\lambda)}{ }_{z}^{*} \mathrm{e}^{i \phi}\right)\right] \\
& -4 e B \sqrt{N N^{\prime}} \varepsilon^{(\lambda)}{ }_{z} \varepsilon^{(\lambda)}{ }_{z}{ }^{*}\left(-r r^{\prime} E E^{\prime}-r r^{\prime} p_{z}{ }^{\prime} p_{z}+E_{\mathrm{o}}{ }^{\prime} E_{\mathrm{o}}\right) \\
& I_{N, N^{\prime}}\left(\omega_{\perp}^{2} / 2 e B\right) I_{N-1, N^{\prime}-1}\left(\omega_{\perp}^{2} / 2 e B\right) \\
& +\left(E E^{\prime}-p_{z}{ }^{\prime} p_{z}+r r^{\prime} E_{\mathrm{o}}{ }^{\prime} E_{\mathrm{o}}\right) \\
& {\left[\varepsilon^{(\lambda)}{ }_{-} \varepsilon^{(\lambda)}{ }_{-}^{*}\left(E_{\mathrm{o}}{ }^{\prime}-r^{\prime} m\right)\left(E_{\mathrm{O}}-r m\right) I_{N, N^{\prime}-1}{ }^{2}\left(\omega_{\perp}{ }^{2} / 2 e B\right)\right.} \\
& \left.+\varepsilon^{(\lambda)}+\varepsilon^{(\lambda)}+{ }^{*}\left(E_{\mathrm{O}}{ }^{\prime}+r^{\prime} m\right)\left(E_{\mathrm{O}}+r m\right) I_{N-1, N^{\prime}}{ }^{2}\left(\omega_{\perp}{ }^{2} / 2 e B\right)\right] \\
& +2 e B \sqrt{N N^{\prime}}\left(r r^{\prime} E E^{\prime}-r r^{\prime} p_{z}{ }^{\prime} p_{z}+E_{\mathrm{o}}{ }^{\prime} E_{\mathrm{o}}\right) \\
& I_{N-1, N^{\prime}}\left(\omega_{\perp}^{2} / 2 e B\right) I_{N, N^{\prime}-1}\left(\omega_{\perp}^{2} / 2 e B\right) \\
& \left.\left(\varepsilon^{(\lambda)}{ }_{-} \varepsilon^{(\lambda)}+{ }^{*} \mathrm{e}^{i 2 \phi}+\varepsilon^{(\lambda)}+\varepsilon^{(\lambda)}{ }_{-}^{*} \mathrm{e}^{-i 2 \phi}\right)\right] \text {. }
\end{aligned}
$$

In the above $\lambda_{c}=h / m c=2.426310^{-10} \mathrm{~cm}$ is the Compton wavelength of the electron. Summing the above expression over the polarization of the photon yields the same result as given by Wunner et al. (1986). 
If we suppose that $\mathrm{e}^{-}$and $\mathrm{e}^{+}$are found in the lowest Landau state $\left(N=N^{\prime}=0\right)$ and $p_{z}+p_{z}{ }^{\prime}=0$, so $\omega \cos \theta=0$, then the photon is emitted perpendicular to the magnetic field $(\theta=\pi / 2)$. We allow any polarization of the photon. Then the above expression for the crosssection reduces to:

$$
\begin{aligned}
& \sigma_{1 \gamma}{ }^{0,0}{ }_{r=-1, r^{\prime}=+1, p_{z}=-p_{z^{\prime}}}=\frac{\alpha{\lambda_{\mathrm{c}}}^{2}}{4\left(B / B_{\mathrm{cr}}\right)} \frac{1}{2\left|p_{z^{\prime}}\right| E_{\mathrm{o}}{ }^{\prime} E E_{\mathrm{o}}} \\
& {\left[\varepsilon_{z} \varepsilon_{z}{ }^{*}\left(E^{\prime} E+p_{z}{ }^{\prime} p_{z}+E_{\mathrm{o}}{ }^{\prime} E_{\mathrm{o}}\right)\right.} \\
& \left.\left(E_{\mathrm{o}}{ }^{\prime}+m\right)\left(E_{\mathrm{o}}+m\right) I_{0,0}{ }^{2}\left(2 E^{2} /\left(m^{2} B / B_{\mathrm{cr}}\right)\right)\right] .
\end{aligned}
$$

Then using Table 2, one obtains:

$$
\begin{aligned}
& \sigma_{1 \gamma, \hat{\varepsilon}^{(2)}}{ }^{0,0}=0 \\
& \sigma_{1 \gamma, \hat{\varepsilon}^{( \pm)}}{ }^{0,0}=\frac{\alpha \lambda_{\mathrm{c}}{ }^{2} m^{2}}{2\left|p_{z}\right| E\left(B / B_{\mathrm{cr}}\right)} \mathrm{e}^{-2 E^{2} /\left(m^{2} B / B_{\mathrm{cr}}\right)} \\
& \sigma_{1 \gamma, \hat{\varepsilon}^{(1)}}{ }^{0,0}=2 \sigma_{1 \gamma, \hat{\varepsilon}^{( \pm)}}{ }^{0,0} \\
& =\sigma_{1 \gamma, \sum_{\lambda} \hat{\varepsilon}^{(\lambda)}} 0,0 .
\end{aligned}
$$

So the radiation obtained in 1 photon annihilation from $\mathrm{e}^{-}$and $\mathrm{e}^{+}$in the ground state with $p_{z}+p_{z}{ }^{\prime}=0$ has linear polarization. The result for summing over polarization agrees with the result of Wunner (1979).

If we take the case of an electron with zero longitudinal momentum $\left(p_{z}=0\right)$, and sum over polarization, then we find:

$\sigma_{1 \gamma}{ }^{0,0}{ }_{p_{z}=0}=\frac{\alpha \lambda_{\mathrm{c}}{ }^{2} m}{\left|p_{z}\right|\left(B / B_{\mathrm{cr}}\right)} \mathrm{e}^{-\left[1+\left(1+\left(p_{z^{\prime}}{ }^{\prime} / m\right)^{2}\right)^{1 / 2}\right]\left(B_{\mathrm{cr}} / B\right)}$

which has been obtained previously by Wunner et al. (1986).

From the conservation laws: $p_{z}+p_{z}{ }^{\prime}=\omega \cos \theta$ and $E+E^{\prime}=\omega$, we find the expression for the longitudinal component of the $\mathrm{e}^{+}$momentum:

$$
\begin{aligned}
& \left.\left(\varepsilon^{(\lambda)}+\varepsilon^{(\lambda)} z^{*} \mathrm{e}^{-i \phi}+\varepsilon^{(\lambda)}{ }_{z} \varepsilon^{(\lambda)}+{ }^{*} \mathrm{e}^{i \phi}\right)\right] \\
& +\sqrt{2 e B N^{\prime}} p_{z}\left[I_{N-1, N^{\prime}-1}\left(\omega_{\perp}{ }^{2} / 2 e B\right) I_{N-1, N^{\prime}}\left(\omega_{\perp}{ }^{2} / 2 e B\right)\right. \\
& \left(\varepsilon^{(\lambda)}+\varepsilon^{(\lambda)} z^{*} \mathrm{e}^{-i \phi}+\varepsilon^{(\lambda)}{ }_{z} \varepsilon^{(\lambda)}+{ }^{*} \mathrm{e}^{i \phi}\right) \\
& +I_{N, N^{\prime}}\left(\omega_{\perp}{ }^{2} / 2 e B\right) I_{N, N^{\prime}-1}\left(\omega_{\perp}{ }^{2} / 2 e B\right) \\
& \left.\left(\varepsilon^{(\lambda)} \varepsilon^{(\lambda)}{ }^{*} \mathrm{e}^{-i \phi}+\varepsilon^{(\lambda)}{ }_{-}^{(\lambda)} z^{*} \mathrm{e}^{i \phi}\right)\right] \\
& -4 e B \sqrt{N N^{\prime}} \varepsilon^{(\lambda)}{ }_{z} \varepsilon^{(\lambda)}{ }_{z}^{*} \\
& I_{N, N^{\prime}}\left(\omega_{\perp}{ }^{2} / 2 e B\right) I_{N-1, N^{\prime}-1}\left(\omega_{\perp}{ }^{2} / 2 e B\right) \\
& +\left(E E^{\prime}-p_{z}{ }^{\prime} p_{z}+m^{2}\right)\left[\varepsilon^{(\lambda)}-\varepsilon^{(\lambda)}{ }^{*} I_{N, N^{\prime}-1}{ }^{2}\left(\omega_{\perp}{ }^{2} / 2 e B\right)\right. \\
& \left.+\varepsilon^{(\lambda)}+\varepsilon^{(\lambda)}+{ }^{*} I_{N-1, N^{\prime}}{ }^{2}\left(\omega_{\perp}{ }^{2} / 2 e B\right)\right] \\
& +2 e B \sqrt{N N^{\prime}} I_{N-1, N^{\prime}}\left(\omega_{\perp}{ }^{2} / 2 e B\right) I_{N, N^{\prime}-1}\left(\omega_{\perp}{ }^{2} / 2 e B\right) \\
& \left.\left(\varepsilon^{(\lambda)}{ }_{-} \varepsilon^{(\lambda)}+{ }^{*} \mathrm{e}^{i 2 \phi}+\varepsilon^{(\lambda)}+\varepsilon^{(\lambda)}{ }^{*} \mathrm{e}^{-i 2 \phi}\right)\right] .
\end{aligned}
$$

If we sum over polarization of the photon in the above equation and take $p_{z}=0$ (i.e. zero longitudinal momentum of the electron) we obtain:

$$
\begin{aligned}
& \sigma_{1 \gamma}{ }^{N, N^{\prime}}=\frac{\alpha \lambda_{\mathrm{c}}{ }^{2} B_{\mathrm{cr}}}{2 B} \frac{1}{p_{z \mathrm{o}}{ }^{\prime} E_{\mathrm{o}}} \frac{\left(1+\delta_{N, 0}\right)}{2} \frac{\left(1+\delta_{N^{\prime}, 0}\right)}{2} \\
& {\left[( E ^ { \prime } E _ { \mathrm { o } } + m ^ { 2 } ) \left[\left(I_{N-1, N^{\prime}-1}^{2}\left(\omega_{\perp}^{2} / 2 e B\right)+I_{N, N^{\prime}}{ }^{2}\right.\right.\right.} \\
& \left.\left(\omega_{\perp}^{2} / 2 e B\right)\right) \sin ^{2} \theta \\
& +\left(I_{N, N^{\prime}-1}^{2}\left(\omega_{\perp}^{2} / 2 e B\right)+I_{N-1, N^{\prime}}{ }^{2}\left(\omega_{\perp}^{2} / 2 e B\right)\right) \\
& \left.\left(1+\cos ^{2} \theta\right)\right] \\
& -2 \sqrt{2 e B N} \omega\left[I_{N, N^{\prime}}\left(\omega_{\perp}^{2} / 2 e B\right) I_{N-1, N^{\prime}}\left(\omega_{\perp}{ }^{2} / 2 e B\right)\right. \\
& \left.+I_{N-1, N^{\prime}-1}\left(\omega_{\perp}{ }^{2} / 2 e B\right) I_{N, N^{\prime}-1}\left(\omega_{\perp}^{2} / 2 e B\right)\right] \sin \theta \cos ^{2} \theta \\
& -4 e B \sqrt{N^{\prime}}\left[I_{N, N^{\prime}}\left(\omega_{\perp}^{2} / 2 e B\right) I_{N-1, N^{\prime}-1}\left(\omega_{\perp}^{2} / 2 e B\right)\right. \\
& \left.\left.+I_{N-1, N^{\prime}}\left(\omega_{\perp}^{2} / 2 e B\right) I_{N, N^{\prime}-1}\left(\omega_{\perp}^{2} / 2 e B\right)\right] \sin ^{2} \theta\right] .
\end{aligned}
$$

This differs by a factor of 2 in the last two terms from Eq. (7) of Wunner et al. (1986), and corrects an error in their expression.

$p_{z}^{\prime}=\frac{\left(E \cos \theta-p_{z}\right)+\sqrt{\left(E \cos \theta-p_{z}\right)^{2}-\sin ^{2} \theta\left[p_{z}^{2}\left(1+\cos ^{2} \theta\right)-4 \gamma\left(N^{\prime}-N\right)-2 E p_{z} \cos \theta\right]}}{\sin ^{2} \theta}$

However, if $\theta=0$ or $180^{\circ}$, the following applies:

$p_{z}{ }^{\prime}=\frac{ \pm 2 \gamma\left(N-N^{\prime}\right) \pm p_{z}^{2}-E p_{z}}{p_{z}+E}$

with the upper sign for $\theta=0$, the lower sign for $180^{\circ}$.

If we average over the polarization of the $\mathrm{e}^{-}$and $\mathrm{e}^{+}$in Eq. (20) above, we obtain:

$$
\begin{aligned}
& \sigma_{1 \gamma}{ }^{N, N^{\prime}}=\frac{\alpha \lambda_{\mathrm{c}}{ }^{2} B_{\mathrm{cr}}}{2 B} \frac{1}{p_{z}{ }^{\prime} E} \frac{\left(1+\delta_{N, 0}\right)}{2} \frac{\left(1+\delta_{N^{\prime}, 0}\right)}{2} \\
& {\left[\varepsilon ^ { ( \lambda ) } { } _ { z } \varepsilon ^ { ( \lambda ) } { } _ { z } ^ { * } ( E ^ { \prime } E + p _ { z } { } ^ { \prime } p _ { z } + m ^ { 2 } ) \left[I_{N-1, N^{\prime}-1}{ }^{2}\right.\right.} \\
& \quad\left(\omega_{\perp}{ }^{2} / 2 e B\right) \\
& \left.+I_{N, N^{\prime}}{ }^{2}\left(\omega_{\perp}{ }^{2} / 2 e B\right)\right] \\
& +p_{z}{ }^{\prime} \sqrt{2 e B N}\left[I_{N-1, N^{\prime}-1}\left(\omega_{\perp}{ }^{2} / 2 e B\right) I_{N, N^{\prime}-1}\left(\omega_{\perp}{ }^{2} / 2 e B\right)\right. \\
& \left(\varepsilon^{(\lambda)} \varepsilon^{(\lambda)}{ }^{*} \mathrm{e}^{-i \phi}+\varepsilon^{(\lambda)}-\varepsilon^{(\lambda)}{ }_{z}^{*} \mathrm{e}^{i \phi}\right) \\
& \quad+I_{N, N^{\prime}}\left(\omega_{\perp}{ }^{2} / 2 e B\right) I_{N-1, N^{\prime}}\left(\omega_{\perp}{ }^{2} / 2 e B\right)
\end{aligned}
$$

\section{Results and discussion}

The decay rate per unit volume is calculated from the cross-section by (e.g. Wunner et al. 1986):

$$
\begin{aligned}
& R_{r, r^{\prime}}{ }^{N, N^{\prime}}\left(p_{z}, p_{z}{ }^{\prime}\right)=\sigma_{r, r^{\prime}}{ }^{N, N^{\prime}}\left(p_{z}, p_{z}{ }^{\prime}\right) n_{\mathrm{e}^{-}}\left(N, p_{z}, r\right) n_{\mathrm{e}^{+}} \\
& \left(N^{\prime}, p_{z}{ }^{\prime}, r^{\prime}\right)\left|p_{z} / E-p_{z}{ }^{\prime} / E^{\prime}\right| .
\end{aligned}
$$

Numerical integration over the distributions for longitudinal momenta of $\mathrm{e}^{-}$and $\mathrm{e}^{+}$yields the total decay rate per unit volume. We use a Gaussian distribution for the longitudinal momentum, $p$, of the electron:

$n(p)=\left(\pi^{1 / 2} \Delta p\right)^{-1} \exp \left[-\left(p-p_{\mathrm{o}}\right)^{2} /(\Delta p)^{2}\right]$

with parameters $\Delta p$, and $p_{\mathrm{o}}$. This is normalized to unity. For the distribution of positron longitudinal momentum, 


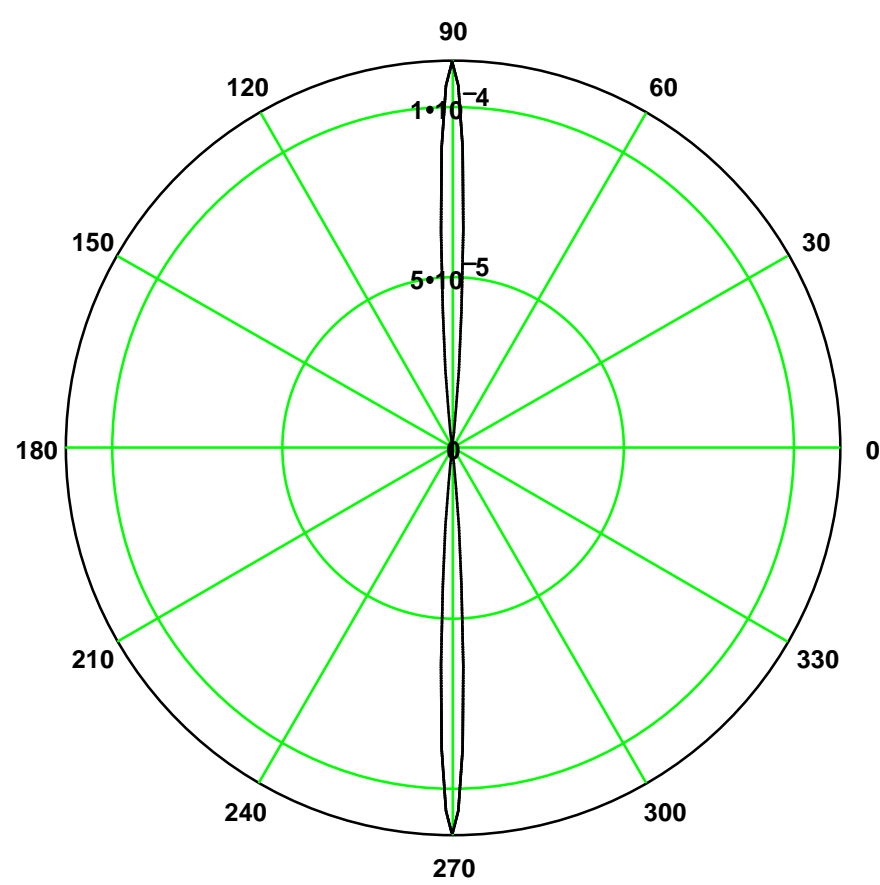

Fig. 1. The $1-\gamma$ annihilation rate as a function of photon emission angle for $B=110^{13}$ Gauss

$q$, we also use the Gaussian distribution, but with parameters $\Delta q$, and $q_{\mathrm{o}}$. The resulting decay rate is then per unit volume, per unit density of positrons, per unit density of electrons.

In order to carry out the integration over the longitudinal momentum distributions, we use the relation giving $p_{z}$ for the electron from the conservation laws, which is:

$$
\begin{aligned}
& p_{z}=\frac{1}{2 \omega \sin ^{2} \theta}\left[\cos \theta\left[\omega^{2} \sin ^{2} \theta+4 \gamma\left(N-N^{\prime}\right)\right]+\right. \\
& {\left[\cos ^{2} \theta\left[\omega^{2} \sin ^{2} \theta+4 \gamma\left(N-N^{\prime}\right)\right]^{2}+\sin ^{2} \theta\left[16 \gamma^{2}\left(N-N^{\prime}\right)^{2}\right.\right.} \\
& -8 \gamma \omega^{2}\left(N-N^{\prime}\right)\left(1+\cos ^{2} \theta\right)+\omega^{4}\left(1-\cos ^{2} \theta\right)^{2}-4 \omega^{2} \\
& \left.\left.\left(m^{2}+4 \gamma N^{\prime}\right)\right]^{1 / 2}\right] .
\end{aligned}
$$

If $N=N^{\prime}=0$, this simplifies to:

$p_{z}=\frac{\omega \sin (2 \theta) / 2+\sqrt{\omega^{2} \sin ^{2} \theta-4 m^{2}}}{2 \sin \theta}$

with the positron longitudinal momentum given by $p_{z}{ }^{\prime}=$ $\omega \cos \theta-p_{z}$, and $\omega$ satisfies the condition $\omega>2 m / \sin \theta$.

For the specific cases presented here for the annihilation rate, we take the electron and positron distributions to be the same. We take the case $p_{\mathrm{o}}=q_{\mathrm{o}}=0$ and $\Delta p=\Delta q=50 \mathrm{keV}, N=N^{\prime}=0$, and unpolarized radiation. Figure 1 shows the annihilation rate as a function of photon emission angle for $B=110^{13}$ Gauss. Figure 2 shows the same but for $B=B_{\mathrm{cr}}$. Figure 1 is consistent with the result of Daugherty \& Bussard (1980) (however we give, in addition, the value of the decay rate): the radiation is confined to a narrow beam centered at $\theta=\pi / 2$ for low magnetic field and small $\Delta p$. Figure 2 shows that the annihilation rate increases rapidly with increasing $B$ (a factor of $\sim 200$ for an increase in $B$ by a factor of 4.4 )

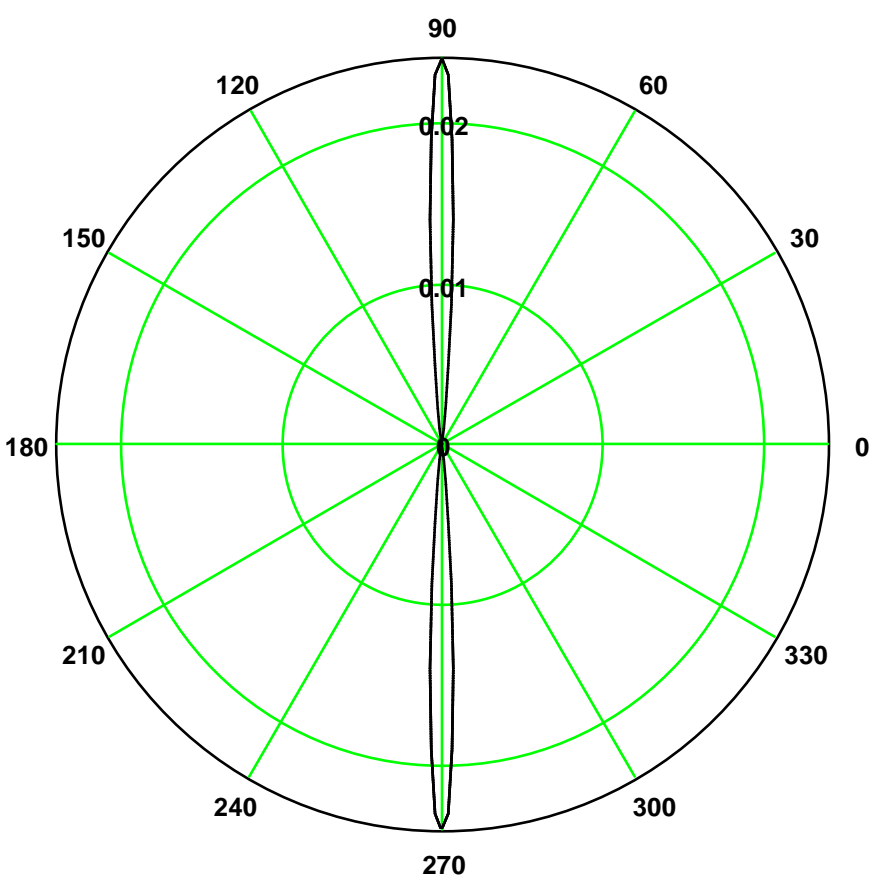

Fig. 2. The $1-\gamma$ annihilation rate as a function of photon emission angle for $B=B_{\text {crit }}=4.41410^{13}$ Gauss

but that the radiation remains well beamed. We find that the beam is narrow for small $\Delta p(<<m c)$ and wide for large $\Delta p(\geq m)$, for all values of $B$.

Next we show some results of numerical evaluations of the cross-sections. First we present cross-sections as a function of magnetic field for the different photon polarizations. The non-relativistic free-space $2 \gamma$ annihilation cross-section for $\mathrm{e}^{-} \mathrm{e}^{+}$pairs with opposite spins and for the electron at rest (e.g. Wunner et al. 1986) is: $\sigma_{\mathrm{o}}=2 \pi \alpha^{2} / \lambda_{\mathrm{c}}{ }^{2} / v^{\prime}$, with $v^{\prime}$ the velocity of the positron. We present our cross-sections normalized by the nonrelativistic free-space $2 \gamma$ annihilation cross-section. The expression for $v^{\prime}$ valid for also for a relativistic positron and in the positron in any Landau state is: $\left.v^{\prime}=p_{z}^{\prime} / \sqrt{(}\left(m^{2}\right)+\left(p_{z}^{\prime 2}\right)+2 N^{\prime} e B\right)$. We note that our calculations of the normalized cross-sections for the cases given in Wunner et al. (1986) agree with their calculations.

For the case of $p_{z}=p_{z}^{\prime}$ and electron with spin down and positron with spin up, the following relations can be shown to hold: $\sigma_{1 \gamma, \hat{\varepsilon}^{(1)}}=2 \sigma_{1 \gamma, \hat{\varepsilon}( \pm)}$ and $\sigma_{1 \gamma, \hat{\varepsilon}^{(2)}}=0$. For $N=N^{\prime}=0$, the above is valid for any values of $p_{z}$ and $p_{z}^{\prime}$.

Figure 3 gives the annihilation cross-section, normalized to $\sigma_{\mathrm{o}}$, for $\mathrm{e}^{-}$with $N=0$ and $r=-1$ (spin down), and $\mathrm{e}^{+}$with $N^{\prime}=0$ and $r^{\prime}=+1$ ( $\operatorname{spin}$ up). For all cases the electron has $p_{z}=0$. The positron has $p_{z}^{\prime} \simeq 0$ (in practise we use a small value, like $10^{-20} \mathrm{MeV} / \mathrm{c}$ to numerically evaluate the cross-section) in one case (with subscript 0 ), and $p_{z}^{\prime}=2 m c$ for the second case (with subscript $2 m c$ ). Since $\sigma_{1 \gamma, \hat{\varepsilon}^{(1)}}=2 \sigma_{1 \gamma, \hat{\varepsilon}^{( \pm)}}$and $\sigma_{1 \gamma, \hat{\varepsilon}^{(2)}}=0$, only the 


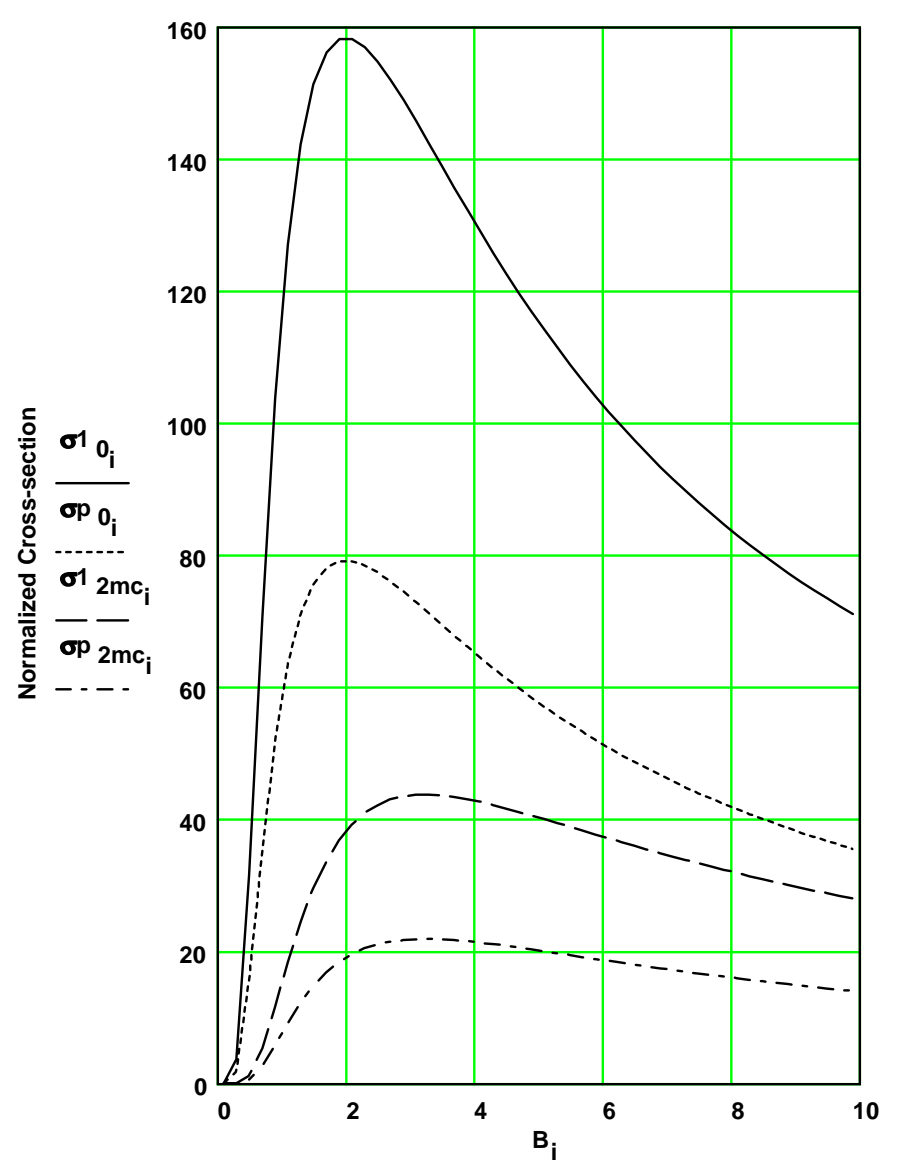

Fig. 3. The annihilation cross-section normalized to the freespace 2-photon nonrelativistic cross-section, $\sigma_{\mathrm{o}}$, for $\mathrm{e}^{-}$with $N=0$ and $r=-1$ (spin down), and $\mathrm{e}^{+}$with $N^{\prime}=0$ and $r^{\prime}=+1$ (spin up). For all cases $p_{z}=0 . \sigma 1_{0}$ is for photon polarization $\hat{\varepsilon}^{(1)}$ and $p_{z}^{\prime} \simeq 0$ and is equal to the total unpolarized cross-section since the $\hat{\varepsilon}^{(2)}$ cross-section is zero, $\sigma p_{0}\left(=\sigma 1_{0} / 2\right)$ is for $\hat{\varepsilon}^{(+)}$and $p_{z}^{\prime} \simeq 0$ (and is equal to that for the $\hat{\varepsilon}^{(-)}$case). $\sigma 1_{2} m c$ is for $\hat{\varepsilon}^{(1)}$ and $p_{z}^{\prime}=2 m c$ and is equal to the total unpolarized cross-section since the $\hat{\varepsilon}^{(2)}$ cross-section is zero, $\sigma p_{2 m c}\left(=\sigma 1_{2 m c} / 2\right)$ is for $\hat{\varepsilon}^{(+)}$and $p_{z}^{\prime}=2 m c$, (and is equal to that for the $\hat{\varepsilon}^{(-)}$case)

cross-sections for photon polarizations $\hat{\varepsilon}^{(1)}$ (labelled $\sigma 1$ ) and $\hat{\varepsilon}^{(+)}$(labelled $\sigma p$ ) are plotted. The cross-sections for unpolarized radiation (sum over polarization for outgoing states) are not plotted, since they are equal to the polarization $\hat{\varepsilon}^{(1)}$ cross-sections in this case.

We find that the cross-sections rise as a function of $B$ rapidly to a peak at $B=\sim 2-3 B_{\text {cr }}$ and decrease slowly for larger $B$. The peak values are larger than the nonrelativistic free-space annihilation cross-section by factors of $\sim 20$ to $\sim 160$. For nonrelativistic parallel momentum, $p_{z}^{\prime} \simeq 0$, one has: for unpolarized radiation or for polarization $\hat{\varepsilon}^{(1)}, \sigma_{1 \gamma}>\sigma_{\mathrm{o}}$ for $B>0.245 B_{\mathrm{cr}}$; and for radiation polarization $\hat{\varepsilon}^{(+)}$or $\hat{\varepsilon}^{(-)}, \sigma_{1 \gamma}>\sigma_{\mathrm{o}}$ for $B>0.245 B_{\mathrm{cr}}$. For large parallel momentum $(>m c)$, the annihilation rate is suppressed compared to the small parallel momentum case. For a relativistic parallel momentum of $p_{z}^{\prime}=2 \mathrm{mc}$,

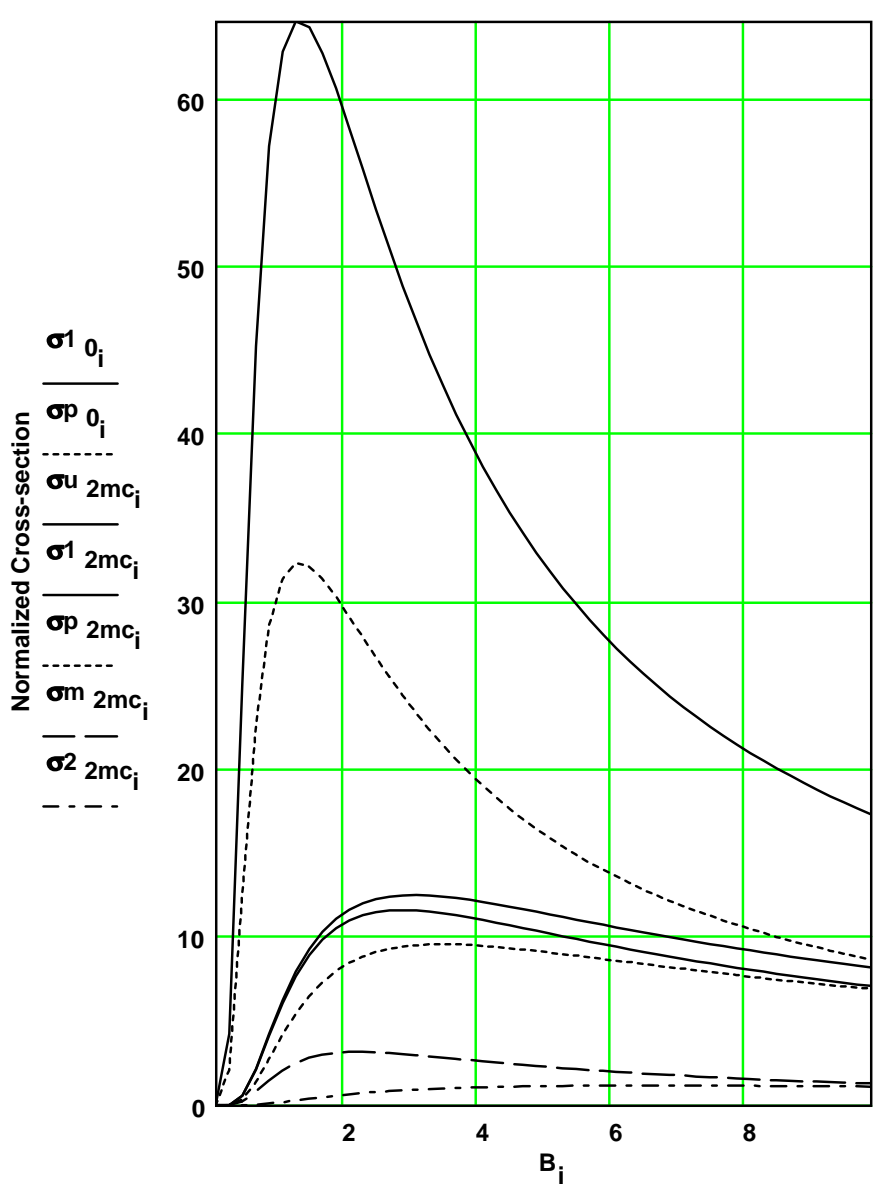

Fig. 4. The annihilation cross-section normalized to $\sigma_{\mathrm{o}}$ for $\mathrm{e}^{-}$ with $N=1$ and $r=-1$ (spin down), and $\mathrm{e}^{+}$with $N^{\prime}=0$ and $r^{\prime}=+1$ (spin up). For all cases $p_{z}=0 . \sigma 1_{0}$ is for photon polarization $\hat{\varepsilon}^{(1)}$ and $p_{z}^{\prime} \simeq 0$ and is equal to the total unpolarized cross-section since the $\hat{\varepsilon}^{(2)}$ cross-section is zero, $\sigma p_{0}\left(=\sigma 1_{0} / 2\right)$ is for $\hat{\varepsilon}^{(+)}$and $p_{z}^{\prime} \simeq 0$ (and is equal to that for the $\hat{\varepsilon}^{(-)}$case). $\sigma u_{2} m c$ is the total unpolarized cross-section for $p_{z}^{\prime}=2 m c, \sigma 1_{2} m c$ is for $\hat{\varepsilon}^{(1)}$ and $p_{z}^{\prime}=2 m c, \sigma p_{2} m c$ is for $\hat{\varepsilon}^{(+)}$ and $p_{z}^{\prime}=2 m c, \sigma m_{2} m c$ is for $\hat{\varepsilon}^{(-)}$and $p_{z}^{\prime}=2 m c$, and $\sigma 2_{2} m c$ is for $\hat{\varepsilon}^{(2)}$ and $p_{z}^{\prime}=2 m c$

one has: for unpolarized radiation or for polarization $\hat{\varepsilon}^{(1)}$, $\sigma_{1 \gamma}>\sigma_{\mathrm{o}}$ for $B>0.484 B_{\mathrm{cr}}$; and for radiation polarization $\hat{\varepsilon}^{(+)}$or $\hat{\varepsilon}^{(-)}, \sigma_{1 \gamma}>\sigma_{\mathrm{o}}$ for $B>0.552 B_{\mathrm{cr}}$. For $p_{z}^{\prime}=4 m c$, one has: for unpolarized radiation or for polarization $\hat{\varepsilon}^{(1)}$, $\sigma_{1 \gamma}>\sigma_{\mathrm{o}}$ for $B>0.950 B_{\mathrm{cr}}$; and for radiation polarization $\hat{\varepsilon}^{(+)}$or $\hat{\varepsilon}^{(-)}, \sigma_{1 \gamma}>\sigma_{\mathrm{o}}$ for $B>1.132 B_{\mathrm{cr}}$.

Figure 4 gives the cross-section normalized to $\sigma_{\mathrm{o}}$ for the same cases as for Fig. 3, except that the electron is now in an excited Landau level $\left(\mathrm{e}^{-}\right.$with $\left.N=1\right)$. The positron has $p_{z}^{\prime} \simeq 0$ in one case (with subscript 0 ), and $p_{z}^{\prime}=2 m c$ for the second case (with subscript $2 m c$ ). For $p_{z}^{\prime} \simeq 0$, one has $\sigma_{1 \gamma, \hat{\varepsilon}^{(1)}}=2 \sigma_{1 \gamma, \hat{\varepsilon}( \pm)}$ and $\sigma_{1 \gamma, \hat{\varepsilon}^{(2)}}=0$, so only the cross-sections for photon polarizations $\hat{\varepsilon}^{(1)}$ (labelled $\sigma 1$ ) and $\hat{\varepsilon}^{(+)}$(labelled $\sigma p$ ) are plotted. The cross-section for unpolarized radiation (sum over polarization for outgoing states) for $p_{z}^{\prime} \simeq 0$ is equal to the 


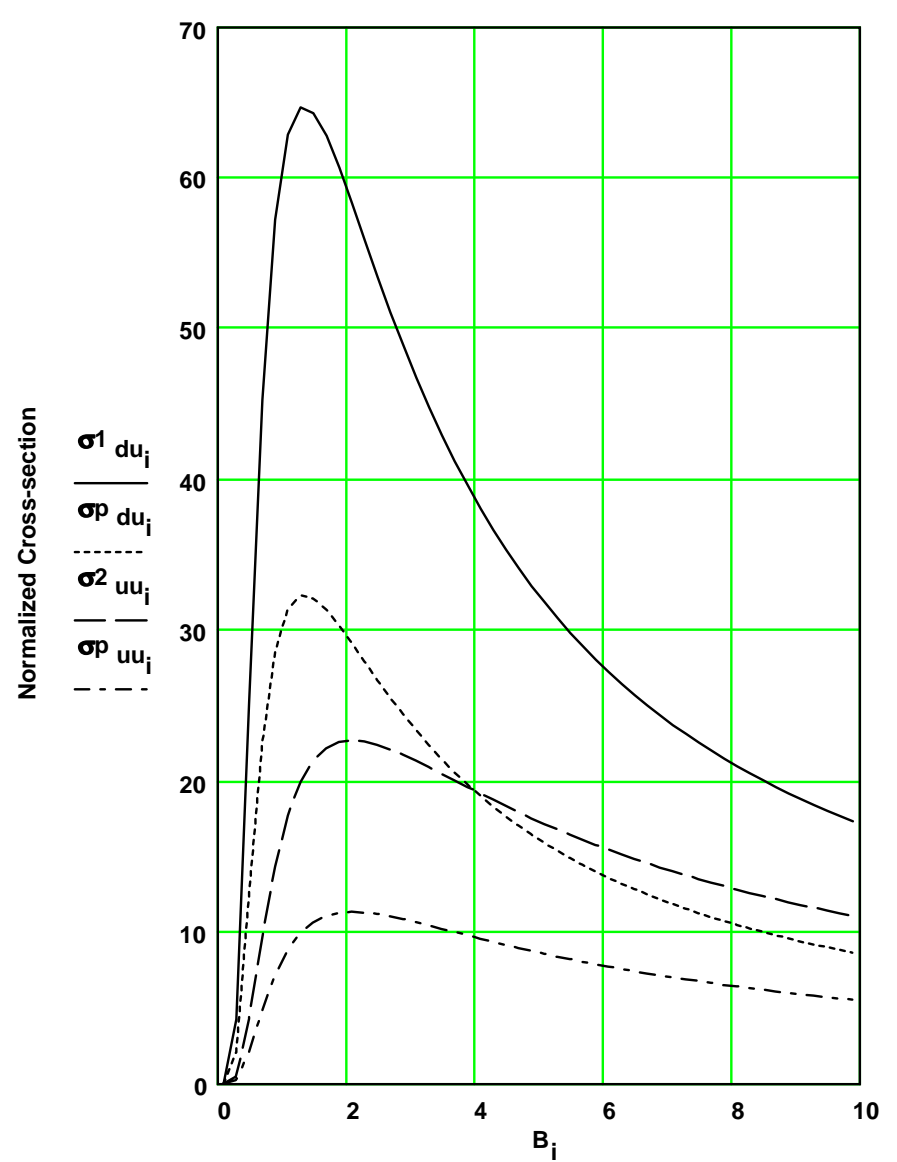

Fig. 5. The annihilation cross-section normalized to $\sigma_{\mathrm{o}}$ for $\mathrm{e}^{-}$ with $N=1$, and $\mathrm{e}^{+}$with $N^{\prime}=0$. For all cases $p_{z}=0$ and $p_{z}^{\prime} \simeq 0 . \sigma 1_{d u}$ is for photon polarization $\hat{\varepsilon}^{(1)}$ and $r=-1$ (spin down) and $r^{\prime}=+1$ (spin up) (and is equal to the total unpolarized cross-section since the $\hat{\varepsilon}^{(2)}$ cross-section is zero). $\sigma p_{d u}\left(=\sigma 1_{d u} / 2\right)$ is for $\hat{\varepsilon}^{(+)}$and $r=-1$ (spin down) and $r^{\prime}=+1$ (spin up), (and is equal to that for the $\hat{\varepsilon}^{(-)}$case). $\sigma 2_{u u}$ is for $\hat{\varepsilon}^{(2)}$ and $r=+1$ (spin up) and $r^{\prime}=+1$ (spin up) (and is equal to the total unpolarized cross-section since the $\hat{\varepsilon}^{(1)}$ cross-section is zero). $\sigma p_{u u}\left(=\sigma 2_{u u} / 2\right)$ is for $\hat{\varepsilon}^{(+)}$and $r=+1$ (spin up) and $r^{\prime}=+1$ (spin up) (and is equal to that for the $\hat{\varepsilon}^{(-)}$case)

polarization $\hat{\varepsilon}^{(1)}$ cross-section. However for nonzero positron momentum (here $p_{z}^{\prime}=2 \mathrm{mc}$ ), the cross-sections for the different polarizations are all different. The cross-section for unpolarized radiation (labelled $\sigma u$ ) is the largest of the 5 curves that peak below a value of 13 , and in decreasing order follow $\sigma 1, \sigma p, \sigma m$ (for photon polarization $\left.\hat{\varepsilon}^{(-)}\right)$, and $\sigma 2$ (for polarization $\hat{\varepsilon}^{(2)}$ ). The $N=1, N^{\prime}=0$ case has lower (unpolarized radiation) cross-sections by a factor of $2.5-3$ compared to the $N=0$ case. However it looks very different in $\hat{\varepsilon}^{(2)}$ radiation, which is not allowed in the $N=0, N^{\prime}=0$ case. $\sigma_{1 \gamma} / \sigma_{\mathrm{o}}$ reaches a maximum as a function of $B$. The maximum value and the $B$ of maximum depend on $N$ and $N^{\prime}$. E.g. for $N=1$ and $N^{\prime}=0$ (Fig. 4), the maximum value is 64.7 , at $B=1.33 B_{\mathrm{cr}}$.

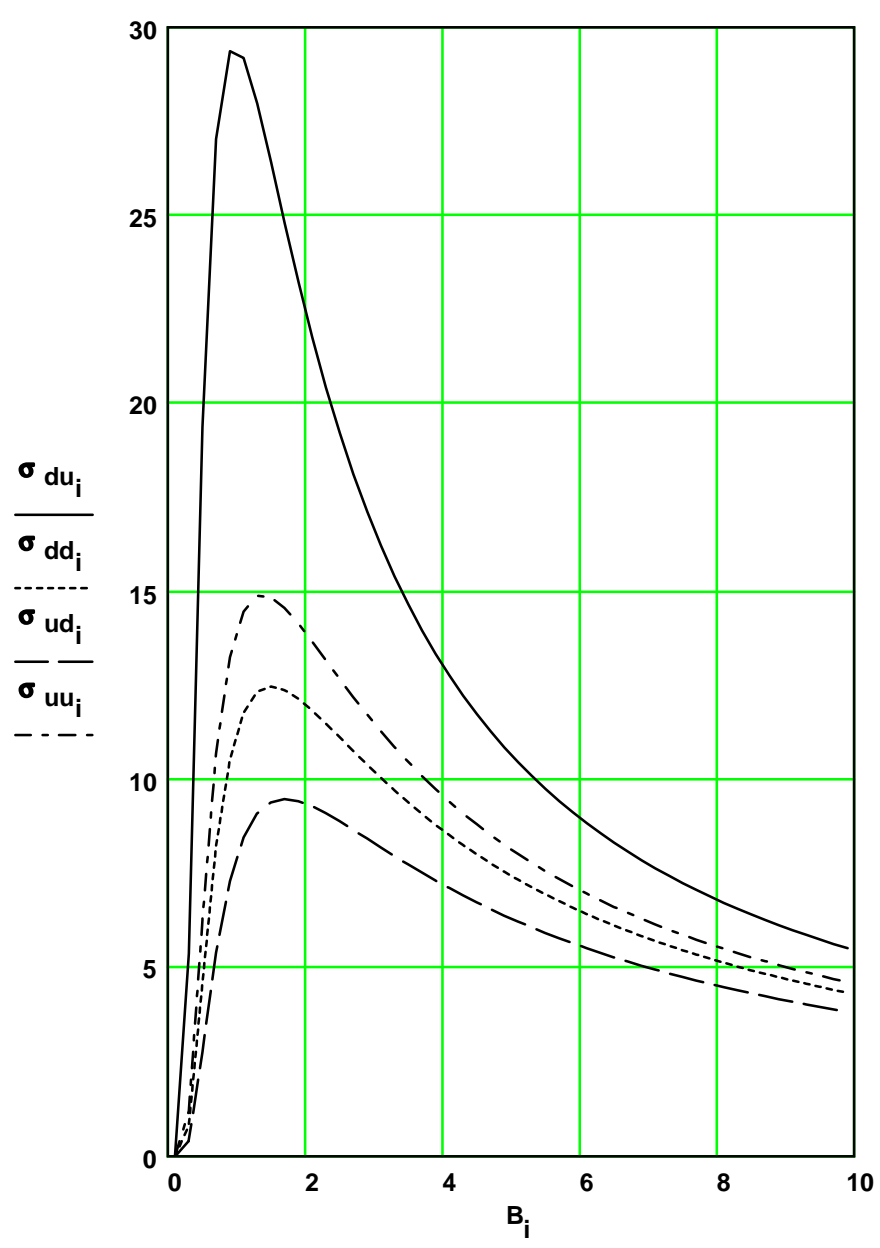

Fig. 6. The annihilation cross-section normalized to $\sigma_{\mathrm{o}}$ for $\mathrm{e}^{-}$ with $N=2$, and $\mathrm{e}^{+}$with $N^{\prime}=1$. For all cases $p_{z}=0$ and $p_{z}^{\prime} \simeq 0$ and the cross-section for unpolarized radiation is plotted. $\sigma_{d u}$ is for $r=-1$ (spin down) and $r^{\prime}=+1$ (spin up). $\sigma_{u u}$ is for $r=+1$ (spin up) and $r^{\prime}=+1$ (spin up). $\sigma_{d d}$ is for $r=-1$ (spin down) and $r^{\prime}=-1$ (spin down). $\sigma_{u d}$ is for $r=+1$ (spin up) and $r^{\prime}=-1$ (spin down)

The cross-section decreases with increasing longitudinal momentum. E.g. for $p_{z}{ }^{\prime}=2 m c$ and $N=1, N^{\prime}=0$ (Fig. 4), the cross-section at $B=0.1 B_{\text {cr }}$ is $1.0510^{6}$ times smaller than for $p_{z}{ }^{\prime} \simeq 0$, and the cross-section at $B=B_{\text {cr }}$ is 11.4 times smaller. From Figs. 3 and 4 , it can be seen that the reduction in cross-section due to larger $p_{z}{ }^{\prime}$ is smaller for larger $B$. A second effect of larger $p_{z}{ }^{\prime}$ is that the cross-section reaches maximum at larger $B$, and, for non-zero $p_{z}{ }^{\prime}$, the $B$ of maximum depends on polarization. E.g., for $p_{z}{ }^{\prime}=2 m c N=1, N^{\prime}=0$ (Fig. 4): the maximum of $\sigma_{1 \gamma} / \sigma_{\mathrm{o}}$ has value at the magnetic field of maximum as follows: 12.5 at $B=3.07 B_{\mathrm{cr}}$ for sum over polarization; 11.6 at $B=2.89 B_{\text {cr }}$ for polarization $\hat{\varepsilon}^{(1)} ; 1.17$ at $B=6.63 B_{\text {cr }}$ for polarization $\hat{\varepsilon}^{(2)} ; 9.60$ at $B=3.48 B_{\text {cr }}$ for polarization $\hat{\varepsilon}^{(+)}$; and 3.18 at $B=2.23 B_{\text {cr }}$ for polarization $\hat{\varepsilon}^{(-)}$. For all values of $B$ and $r=-1, r^{\prime}=+1$ one has: $\sigma_{1 \gamma, \hat{\varepsilon}^{(1)}}>\sigma_{1 \gamma, \hat{\varepsilon}^{(+)}}>\sigma_{1 \gamma, \hat{\varepsilon}^{(-)}}>\sigma_{1 \gamma, \hat{\varepsilon}^{(2)}}$. 


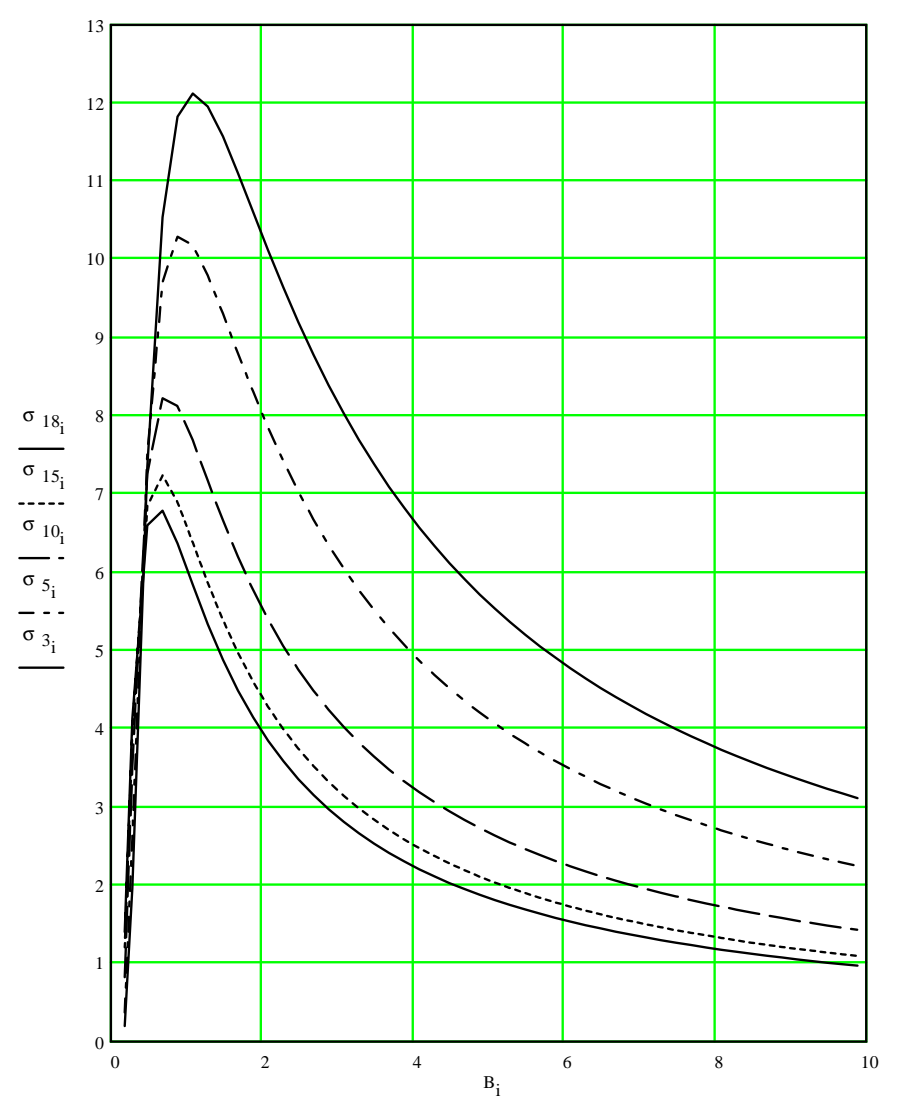

Fig. 7. The annihilation cross-section normalized to $\sigma_{\mathrm{o}}$ as a function of $N$ and $N^{\prime}$. For all cases $p_{z}=p_{z}^{\prime}=2 m c, \mathrm{e}^{-}$ has $r=-1$ (spin down) and $\mathrm{e}^{+}$has $r^{\prime}=+1$ (spin up), and the cross-section for unpolarized radiation is plotted. $\sigma_{18}$ is for $N=N^{\prime}=18 . \sigma_{15}$ is for $N=N^{\prime}=15 . \sigma_{10}$ is for $N=N^{\prime}=10$. $\sigma_{5}$ is for $N=N^{\prime}=5 . \sigma_{3}$ is for $N=N^{\prime}=3$

We next compare the cross-sections for different spin states of the electron. In Fig. 5, the normalized crosssection is presented for two different spin states: $\mathrm{e}^{-}$with $N=1, p_{z}=0$ and $r= \pm 1$, and $\mathrm{e}^{+}$with $N^{\prime}=0, p_{z}{ }^{\prime} \simeq 0$ and $r^{\prime}=+1$. The upper two curves ( $\sigma 1$ for photon polarization $\hat{\varepsilon}^{(1)}$ and $\sigma p$ for polarization $\left.\hat{\varepsilon}^{(+)}\right)$are for $r=-1$, $r^{\prime}=+1$ (denoted by subscript $\mathrm{du}$ in the figure) and are the same as for Fig. 4. The lower two curves $(\sigma 2$ for photon polarization $\hat{\varepsilon}^{(2)}$ and $\sigma p$ for polarization $\left.\hat{\varepsilon}^{(+)}\right)$are for $r=+1, r^{\prime}=+1$ (denoted by subscript $u u$ ). Here and in what follows the first arrow indicates whether the electron spin is up or down and the second arrow indicates whether the positron spin is up or down (for the figures we use $u$ and $d$ to indicate spin up or down). For the case of $\downarrow \uparrow$ we have $\sigma_{1 \gamma, \hat{\varepsilon}(1)}=2 \sigma_{1 \gamma, \hat{\varepsilon}( \pm)}$ and $\sigma_{1 \gamma, \hat{\varepsilon}^{(2)}}=0$. However for the case of electron and positron both spin-up $(\uparrow \uparrow)$, we have $\sigma_{1 \gamma, \hat{\varepsilon}^{(2)}}=2 \sigma_{1 \gamma, \hat{\varepsilon}^{( \pm)}}$and $\sigma_{1 \gamma, \hat{\varepsilon}^{(1)}}=0$. The cross-section for spins $\downarrow \uparrow$ is larger than that for spins $\uparrow \uparrow$ (e.g. by factor 22.0 at $B=0.1 B_{\mathrm{cr}}$ ), the difference decreasing with increasing $B$ (e.g. by factor 1.56 at $B=9.9 B_{\mathrm{cr}}$ ).

Generally for $N>0$ and $N^{\prime}>0$ (so both spins are allowed for positron and electron) and if $p_{z}=p_{z}^{\prime}$, one

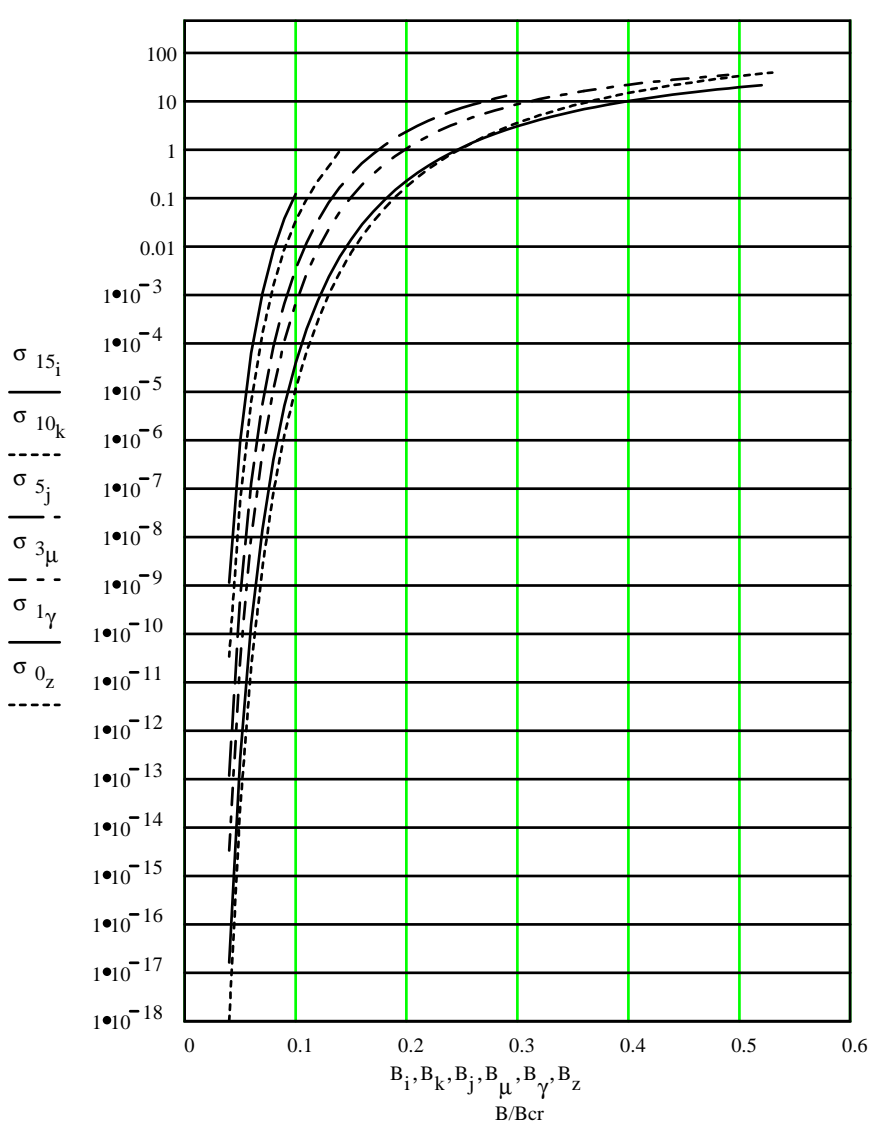

Fig. 8. The annihilation cross-section normalized to $\sigma_{\mathrm{o}}$ as a function of $N$ and $N^{\prime}$. For all cases the total energy of the electron and positron are each $2 m c$, the cross-section is for average over electron and positron spins, and the cross-section is for unpolarized radiation. $\sigma_{15}$ is for $N=N^{\prime}=15 . \sigma_{10}$ is for $N=N^{\prime}=10 . \sigma_{5}$ is for $N=N^{\prime}=5 . \sigma_{3}$ is for $N=N^{\prime}=3$. $\sigma_{1}$ is for $N=N^{\prime}=1$. $\sigma_{0}$ is for $N=N^{\prime}=0$. For each curve (except $N=N^{\prime}=0$ ) there is a maximum B, for which the parallel energy drops to zero

has the following relation for the cross-sections for different photon polarizations: For the cases with antiparallel spins $(\downarrow \uparrow$ or $\uparrow \downarrow)$ we have $\sigma_{1 \gamma, \hat{\varepsilon}^{(1)}}=2 \sigma_{1 \gamma, \hat{\varepsilon}^{( \pm)}}$and $\sigma_{1 \gamma, \hat{\varepsilon}^{(2)}}=0$. For the cases with parallel spins ( $\downarrow \downarrow$ or $\left.\uparrow \uparrow\right)$ we have $\sigma_{1 \gamma, \hat{\varepsilon}^{(2)}}=2 \sigma_{1 \gamma, \hat{\varepsilon}( \pm)}$ and $\sigma_{1 \gamma, \hat{\varepsilon}^{(1)}}=0$. In the above two cases we have the unpolarized cross-section is the same as the $\hat{\varepsilon}^{( \pm)}$cross-sections. For summing over polarization we have for all $B: \sigma_{1 \gamma, \downarrow \uparrow}>\sigma_{1 \gamma, \uparrow \uparrow}>\sigma_{1 \gamma, \downarrow \downarrow}>\sigma_{1 \gamma, \uparrow \downarrow}$. Figure 6 shows the normalized cross-section for unpolarized radiation and for four different spin states: $\mathrm{e}^{-}$with $N=2$, $p_{z}=0$ and $r= \pm 1$, and $\mathrm{e}^{+}$with $N^{\prime}=1, p_{z}{ }^{\prime} \simeq 0$ and $r^{\prime}= \pm 1$. The cross-section for $r=-1, r^{\prime}=1$ is denoted $\sigma_{d u}$, for $r=1, r^{\prime}=1$ is denoted $\sigma_{u u}$, for $r=-1, r^{\prime}=-1$ is denoted $\sigma_{d d}$, and for $r=1, r^{\prime}=-1$ is denoted $\sigma_{u d}$.

Next we consider the dependence of the cross-section on $N$ and $N^{\prime}$. Figure 7 shows the normalized cross-section for $\mathrm{e}^{-}, \mathrm{e}^{+}$spins $\downarrow \uparrow$, summed over photon polarization, for five sets of values of $\left(N, N^{\prime}\right):(3,3),(5,5),(10,10)$, $(15,15)$ and $(18,18)$. Here we have taken the case of 
longitudinal momentum: $p_{z}=p_{z}{ }^{\prime}=2 \mathrm{mc}$. (The case of zero longitudinal momentum and sum over polarization has been considered by Wunner et al. 1986.) Since $p_{z}=p_{z}{ }^{\prime}$ the relations in the last paragraph above give the cross-sections for the individual polarizations. I.e., $\sigma_{1 \gamma, \hat{\varepsilon}^{(1)}}=2 \sigma_{1 \gamma, \hat{\varepsilon}^{( \pm)}}$and $\sigma_{1 \gamma, \hat{\varepsilon}^{(2)}}=0$. In Fig. 7, the number label on $\sigma$ represents the values of $N$ and $N^{\prime}$. For small $B$ $\left(<B_{\mathrm{cr}}\right)$, the cross-sections at first increase with $N$ and $N^{\prime}$ then reach a maximum and decrease for larger $N$ and $N^{\prime}$. E.g. for $p_{z}=p_{z}{ }^{\prime}=2 m c$, and $B=0.12 B_{\mathrm{cr}}\left(=5.3010^{12}\right.$ Gauss), the maximum cross-section is for $N^{\prime}=N=12$,

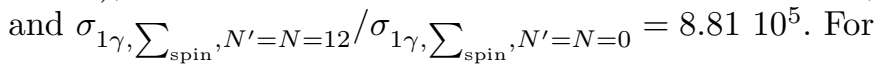
large $B\left(>B_{\mathrm{cr}}\right)$, one finds: $\sigma_{1 \gamma}$ is largest for for $N^{\prime}=$ $N=0$; and $\sigma_{1 \gamma}$ for $N^{\prime}=N$ decreases monotonically with increasing $N$.

Generally we have the following relations for the relative size of the cross-sections for different electron spins at fixed $N$ and $N^{\prime}$. (a) For $N^{\prime}=N, \sigma_{1 \gamma, \downarrow \uparrow}>\sigma_{1 \gamma, \uparrow \uparrow}=$ $\sigma_{1 \gamma, \downarrow \downarrow}>\sigma_{1 \gamma, \uparrow \downarrow}$ (except for $N^{\prime}=N=0$, where we only have the possibility for spins of $\downarrow \uparrow$ ). (b) For $N^{\prime}<N$, $\sigma_{1 \gamma, \downarrow \uparrow}>\sigma_{1 \gamma, \uparrow \uparrow}>\sigma_{1 \gamma, \downarrow \downarrow}>\sigma_{1 \gamma, \uparrow \downarrow}$ (except for $N^{\prime}=0$, where we only have the possibilities for spins of $\uparrow \uparrow$ and $\downarrow \uparrow)$. (c) For $N^{\prime}>N, \sigma_{1 \gamma, \downarrow \uparrow}>\sigma_{1 \gamma, \downarrow \downarrow}>\sigma_{1 \gamma, \uparrow \uparrow}>\sigma_{1 \gamma, \uparrow \downarrow}$ (except for $N=0$, where we only have the possibilities for spins of $\downarrow \uparrow$ and $\downarrow \downarrow)$.

Finally, we show a set of cross-sections for different $N$ and $N^{\prime}$ (with $\left.N^{\prime}=N\right)$, but rather than at fixed $p_{z}$ and $p_{z}^{\prime}$, they are at fixed $E_{\mathrm{e}-}$ and $E_{\mathrm{e}+}$. In this case, because the $\mathrm{e}^{-}\left(\right.$or $\left.\mathrm{e}^{+}\right)$energy increases with $B$ for $N>0$ (or $N^{\prime}>0$ ), there is a maximum $B$ at constant $E_{\mathrm{e}-\text { for }} N>0$ (or at constant $E_{\mathrm{e}+}$ for $N^{\prime}>0$ ). Since we take $E_{\mathrm{e}-}=E_{\mathrm{e}+}$, the maximum $B$ is the same for both $\mathrm{e}^{-}$and $\mathrm{e}^{-}$. Figure 8 shows the resulting normalized cross-sections as a function of $B / B_{\mathrm{cr}}$ for six sets of values of $\left(N, N^{\prime}\right)$ : $(0,0)$, $(1,1),(3,3),(5,5),(10,10)$, and $(15,15)$ and for $\mathrm{e}^{-}$ with spin down and $\mathrm{e}^{+}$with spin down and unpolarized radiation. In all cases we have $p_{z}=p_{z}^{\prime}$, so the relation for the different photon polarization holds: $\sigma_{1 \gamma, \hat{\varepsilon}^{(1)}}=2 \sigma_{1 \gamma, \hat{\varepsilon}^{( \pm)}}$ and $\sigma_{1 \gamma, \hat{\varepsilon}^{(2)}}=0$.
In summary, we have presented formulae for the one photon pair annihilation cross-section for different photon polarizations which are valid for any magnetic field and any Landau and spin states of the electron and positron. These results are generalizations of those of Wunner et al. (1986). This is an important generalization, since the new results presented here allow calculation of the polarization of the emitted radiation, which is significantly different from zero, and strongly dependent on the momenta, spin and Landau states of the electron and positron.

Acknowledgements. DAL thanks the Natural Sciences and Engineering Research Council of Canada for support.

\section{References}

Bussard R., Alexander S., Meszaros P., 1986, Phys. Rev. D 34, 440

Daugherty J., Bussard R., 1980, ApJ 238, 296

Graziani C., 1993, ApJ 412, 351

Harding A., 1986, ApJ 300, 167

Harding A., Preece R., 1987, ApJ 319, 939

Herold H., 1979, Phys. Rev. D 19, 2868

Herold H., Ruder H., Wunner G., 1982, A\&AS 115, 90

Kaminker A., Pavlov G., Mamradze P., 1987, Ap\&SS 138, 1

Klepikov N., 1987, JETP 26, 19

Kouveliotou C., et al., 1999, ApJL 510, L115

Latal H., 1986, ApJ 309, 372

Semionova L., 1983, M. Sc. Thesis, University of Costa Rica Semionova L., Leahy D., 1999, Phys. Rev. D, Oct. 1 issue

Sokolov A., Ternov I., Relativistic Electron. Nauka, Moscow, 1983

Vasisht G., Gotthelf E., 1997, ApJ 486, L129

White D., 1974, Phys. Rev. D 9, 868

White D., 1978, Phys. Rev. D 18, 2166

Wunner G., 1979, Phys. Rev. Lett. 42, 8

Wunner G., Paez J., Herold H., Ruder H., 1986, A\&A 170, 179 